\title{
ANÁLISIS COMPARATIVO DEL DISEÑO ESTRUCTURAL DE UNA EDIFICACIÓN REGULAR E IRREGULAR DE OCHO NIVELES EN SISTEMA DE PÓRTICOS APLICANDO LA NORMA E.030 2003, 2016 Y 2018 DISEÑO SISMORRESISTENTE EN LA CIUDAD DE LIMA
}

\section{COMPARATIVE ANALYSIS OF THE STRUCTURAL DESIGN OF A REGULAR AND IRREGULAR BUILDING OF EIGHT LEVELS IN A PORTS SYSTEM APPLYING STANDARD E.030 2003, 2016 AND 2018 SEISMIC RESISTANT DESIGN IN THE CITY OF LIMA}

Jorge Eduardo, De La Cruz Alvarez ${ }^{1}$; Roberto Roland, Yoctun Rios ${ }^{2}$

Recibido 03/09/2021: Aprobado: 19/11/2021

DOI: https://doi.org/10.51372/gacetatecnica231.5

\section{RESUMEN}

Se presenta el análisis comparativo de las normas E.030 2003, 2016 y 2018 diseño sismorresistente en un estudio de estructuras mediante el sistema de pórticos para uso de oficinas en la ciudad de Lima, se centra en la comparación de periodos de vibración, porcentaje de masa participativa, espectro de diseño, distorsión de entrepiso, fuerza cortante basal estática, dinámica y las fuerzas máximas de diseño (momento flector, cortante y axial), modelado mediante el software Etabs 17. La estructura regular presentó variación de 12,50\% de cortante estática y 12,20\% para el análisis dinámico según la norma del 2003 en comparación del 2016 - 2018 y la estructura irregular varia en 50\% según la norma del 2003 en comparación del 2016, así mismo una variación de -16,67\% entre la norma del 2016 y 2018 afectadas directamente por los cambios de irregularidad. La norma del 2018 tiene resultados intermedios considerando periodos modales para ambas estructuras mediante el análisis estático y dinámico.

Palabras clave: norma E.030; análisis comparativo sismorresistente; diseño estructural

\footnotetext{
${ }^{1}$ Jorge Eduardo, De La Cruz Alvarez. Estudiante en Ingeniería Civil Universidad Peruana Unión. Lima, Perú. Correo: jorgedelacruz@upeu.edu.pe ORCID: https://orcid.org/0000-0003-2576-0199

${ }^{2}$ Roberto Roland, Yoctun Rios. Asesor de Investigación Escuela Profesional de Ingeniería Civil. Universidad Peruana Unión. Lima, Perú. Correo: robertoyoctun@upeu.edu.pe ORCID: https://orcid.org/0000-0002-0927$\underline{7829}$
} 


\section{ABSTRACT}

The comparative analysis of the E.030 2003, 2016 and 2018 seismic design standards is presented, in a study of structures using the portal system for office use in the city of Lima, it focuses on the comparison of vibration periods, percentage of participatory mass, design spectrum, mezzanine distortion, static basal shear force, dynamic and maximum design forces (bending, shear and axial moment), modeled using Etabs 17 software. The regular structure presented variation of $12.50 \%$ of static shear and $12.20 \%$ for the dynamic analysis according to the 2003 standard compared to 2016 - 2018 and the irregular structure varies by $50 \%$ according to the 2003 standard compared to 2016, also a variation of $-16.67 \%$ between the 2016 and 2018 norm directly affected by the irregularity changes. The 2018 standard has intermediate results considering modal periods for both structures through static and dynamic analysis.

\section{Keywords: standard E.030; comparative seismic analysis; structural design}

\section{INTRODUCCIÓN}

La norma peruana E.030 diseño sismorresistente ha sido modificada con el paso de los años, la primera data de 1970 y abarcaba las experiencias de los terremotos importantes desde 1940 hasta el 2001, además, el cambio del comité Visión 2000 para obtener la norma del 2003 [1]. Sus reformas continuaron hasta dar con la del 2016 y 2018, en los que por diferentes estudios numéricos se determinó un nuevo mapa de peligro sísmico a diferencia del 2003, la cual se mantiene hasta la actualidad.

En el Perú, específicamente en la ciudad de Lima se tiene mayor probabilidad de generarse un sismo de gran magnitud, en consecuencia, las actualizaciones de la norma sismorresistente E.030 sigue en constante investigación de acuerdo a la zonificación, estructuración, seguridad y economía, por esta razón todos los cambios que se dan en la norma afecta directamente al diseño estructural [2]. Es por ello la importancia de realizar un análisis comparativo y determinar el comportamiento lineal estático y dinámico según las normativas con la ayuda del programa de computación Etabs 17, el cual facilita obtener la variación de las fuerzas estáticas, dinámicas, desplazamientos y del coeficiente de reducción (R) afectada directamente por los cambios de irregularidad.

Por ello se realizó el análisis comparativo evaluando el comportamiento sísmico de una estructura regular y otra irregular de 8 niveles en la ciudad de Lima, considerando las normas especificadas anteriormente [3] [4] y [5]. Además, para las estructuras regulares se determinó la variación que existe en el análisis al considerar los tres periodos que permite la norma, mientras que en la estructura irregular la variación en al análisis estático, debido que la norma no lo permitió. 
ANÁLISIS COMPARATIVO DEL DISEÑO ESTRUCTURAL DE UNA EDIFICACIÓN REGULAR E IRREGULAR DE OCHO NIVELES EN SISTEMA DE PÓRTICOS APLICANDO LA NORMA E.030 2003, 2016 Y 2018 DISEÑO SISMORRESISTENTE EN LA CIUDAD DE LIMA

\section{DESARROLLO}

\subsection{Parámetros de Análisis}

Es indispensable definir los parámetros sísmicos más importantes que incrementan o disminuyen las fuerzas de diseño, de acuerdo al cambio de las normas de diseño sismorresistente E.030 del 2003, 2016 y 2018:

El factor de zonificación (Z) en la Tabla 1, se basa en la distribución espacial de la sismicidad observada y las características generales de los movimientos sísmicos. El factor presente en todos los reglamentos del mundo [6], en el Perú se caracteriza por describir la aceleración que tendría la superficie cada 500 años en suelo firme $S 1$ según la E.030 [4] y [5] de lo que antes se consideraba en roca, ahora para considerarse como tal, al factor $Z$ se multiplicara por 0,8 . Donde se sigue evaluando el peligro sísmico existente prediciendo probabilísticamente las aceleraciones máximas que podrían ocurrir en cualquier parte del país, utilizando leyes de atenuación de aceleraciones y correlacionando la sismicidad y la tectónica para determinar las fuentes sismogénicas y sus respectivos parámetros sismológicos [7].

Tabla 1. Factor de zonificación. Fuente: [3] [4] y [5]

\begin{tabular}{ccccc}
\hline \multicolumn{2}{c}{ Norma 2003 } & \multicolumn{3}{c}{ Norma 2016 y 2018 } \\
\hline Zona & $\mathrm{Z}(\mathrm{g})$ & Zona & $\mathrm{Z}(\mathrm{g})$ & Variación \\
\hline 3 & 0,40 & 4 & 0,45 & $13 \%$ \\
2 & 0,30 & 3 & 0,35 & $17 \%$ \\
1 & 0,15 & 2 & 0,25 & $-17 \%$ \\
& & 1 & 0,10 & $-33 \%$ \\
\hline
\end{tabular}

El factor de suelo $S$, los periodos cortos $T P$ y periodos largos $T L$ según la norma E.030 son valores que describen las condiciones locales, el factor $S$ depende del tipo de suelo y la zona en el 2016 y 2018, así como de una nueva variable $T L$, este se caracteriza por definir el inicio de la zona espectral con desplazamiento constante. Para la zona de Lima los factores de suelo $S$ disminuyeron solo para los suelos blandos y malos, pero los suelos característicos de Lima suelos rígidos $S 1$ la norma del 2016 y 2018 no generó ninguna modificación, de acuerdo al suelo (S1) les corresponde a las tres normas el factor $S=1$ y los periodos $T P=0,4$ s y $T L=$ $2,5 \mathrm{~s}$.

Aksoylu et al. [8] mencionaron la importancia del factor de amplificación sísmica $C$ y presentaron sugerencias para determinar el periodo por Rayleigh o periodo alternativo [3]. Además, que cambia en zonas de alta actividad sísmica donde podría ocurrir eventos de gran magnitud y mide cuánto amplifica la aceleración en la estructura de acuerdo a las características de sitio. En la Tabla 2 se puede observar los cambios y nuevas condiciones de 
las normativas, siendo el periodo de la estructura el parámetro principal del cambio del factor $C$.

Tabla 2. Factor de amplificación sísmica (C). Fuente: [3] [4] y [5]

\begin{tabular}{cccc}
\hline \multicolumn{2}{c}{ Norma 2003 } & \multicolumn{2}{c}{ Norma 2016 y 2018 } \\
\hline Condiciones & $\mathrm{C}$ & Condiciones & $\mathrm{C}$ \\
$C \leq 2,5$ & $2,5 \cdot \frac{T p}{T}$ & $T<T p$ & 2,5 \\
& & $T p<T<T L$ & $C=2,5 \cdot \frac{T p}{T}$ \\
& & $T>T L$ & $C=2,5 \cdot \frac{T p \cdot T l}{T^{2}}$ \\
\hline
\end{tabular}

El factor de importancia $U$ sigue manteniendo el mismo valor de $U=1$ para estructuras comunes, y el coeficiente de reducción $R$ presenta algunas discrepancias con diferentes estructuras; mientras que para la norma del 2003 son estructuras regulares pero muchas de ellas presentan irregularidad en la norma del 2016 y 2018 de acuerdo al sistema estructural y su geometría, definiendo los parámetros que involucran a este factor como el coeficiente básico de reducción $R o$, irregularidad en altura $I a$ e irregularidad en planta $I p$. El coeficiente básico de reducción $R o$ para pórticos no generó ninguna modificación y continua con el valor $R o=8$. Por otro lado, se tienen los cambios de irregularidades en cada actualización de la norma que se presenta en la Tabla 3.

Tabla 3. Irregularidades en planta y en altura. Fuente: [3] [4] y [5]

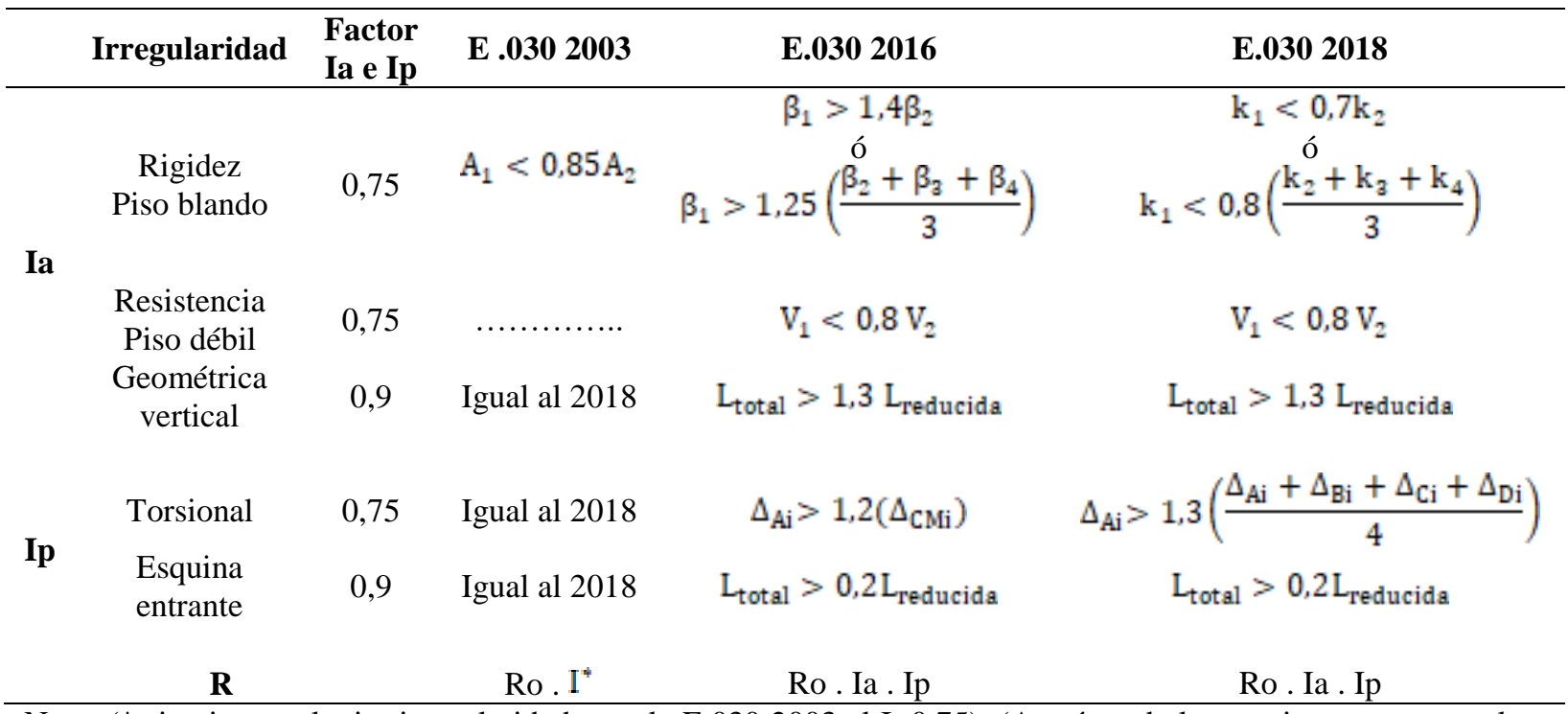

Nota: (* si existe cualquier irregularidad para la E.030 2003 el I=0,75), (A = área de las secciones transversales de los elementos verticales), $(\beta=$ distorsión o deriva de entrepiso $),(\mathrm{V}=$ Fuerza cortante $),\left(\Delta_{A i}=\right.$ máximo desplazamiento relativo de entrepiso en un extremo), $\left(\Delta_{\mathrm{CM}}=\right.$ desplazamiento relativo del centro de masa) y $\left(k_{1}\right.$ = rigidez lateral del entrepiso).

La fuerza cortante basal de la E.030 es la fuerza cortante total que actúa en la base de la estructura, correspondiente a cada dirección de acuerdo al sistema estructural utilizado con los 
ANÁLISIS COMPARATIVO DEL DISEÑO ESTRUCTURAL DE UNA EDIFICACIÓN REGULAR E IRREGULAR DE OCHO NIVELES EN SISTEMA DE PÓRTICOS APLICANDO LA NORMA E.030 2003, 2016 Y 2018 DISEÑO SISMORRESISTENTE EN LA CIUDAD DE LIMA

valores de $Z, U, C, S, R$ y multiplicado por el peso sísmico de la estructura mostrado en la ecuación (1), logrando tener la fuerza cortante basal de la estructura mediante el análisis estático y según se mencionan en otros códigos de diseño con alta actividad sísmica [9].

$$
V=\frac{Z \cdot U \cdot C \cdot S}{R} \cdot P
$$

El método que presenta la E.030 es el análisis dinámico modal espectral sin modificación hasta la norma del 2018, definiendo así la cortante basal mínima que dependerá de su regularidad y el método de combinación cuadrática completa $C Q C$, que considera una correlación entre los valores modales máximos para así obtener valores más probables de desplazamiento y fuerzas [10]. La Tabla 4 muestra la distribución de la fuerza sísmica en altura según el análisis estático, donde inicialmente la E.030 2003 tenía una distribución lineal, en la norma del 2016 y 2018 se eliminó la fuerza concentrada en el último nivel denominada $F a$ y se corrige la distribución lineal con el exponente $k$ relacionado directamente con el periodo fundamental de las estructuras.

Tabla 4. Distribución de fuerza en altura. Fuente: [3] [4] y [5]

\begin{tabular}{|c|c|c|}
\hline & E.030 2003 & E.030 2016 y 2018 \\
\hline \multirow{2}{*}{ Periodos $(\mathrm{T})$} & $T>0,7_{s}$ & $T \leq 0,5_{\text {geg }_{0}} \ldots k=1,0$ \\
\hline & $F_{\alpha}=0,07 . T \cdot V \leq 0.15 . V$ & $T>0,5_{\text {geg }_{0},} k=(0,75+0,5 . T) \leq 2$ \\
\hline $\begin{array}{c}\text { Distribución de } \\
\text { fuerzas (Fi) }\end{array}$ & $F i=\frac{P_{i} \cdot h_{i}}{\sum_{i=1}^{n} P_{j} \cdot h_{j}} \cdot\left(V-F_{a}\right)$ & $\begin{array}{c}F i=\mathrm{\alpha}_{\mathrm{i}} \cdot V \\
\mathrm{\aleph}_{\mathrm{i}}=\frac{P_{\mathrm{i}} \times\left(h_{\mathrm{i}}\right)^{k}}{\sum_{\eta=1}^{n} P_{j} \cdot\left(h_{j}\right)^{k}}\end{array}$ \\
\hline
\end{tabular}

La Tabla 5 muestra los desplazamientos laterales que sufre una estructura después del análisis y sus cambios para estructuras irregulares, mientras las estructuras regulares se han mantenido hasta la norma del 2018.

Tabla 5. Desplazamientos laterales. Fuente: los autores

\begin{tabular}{cccc}
\hline \multicolumn{3}{c}{ Desplazamientos } \\
\hline Estructura & E.030 2003 & E.030 2016 & E.030 2018 \\
Regular & $0,75 \mathrm{R} . \mathrm{d}$ & $0,75 \mathrm{R} . \mathrm{d}$ & $0,75 \mathrm{R} . \mathrm{d}$ \\
Irregular & $0,75 \mathrm{R} . \mathrm{d}$ & $\mathrm{R} . \mathrm{d}$ & $0,85 \mathrm{R} . \mathrm{d}$ \\
\hline
\end{tabular}

Nota: (D= desplazamiento inelástico lateral), $(\mathrm{d}=$ desplazamiento elástico lateral), ( $\mathrm{R}=$ coeficiente de reducción). Fuente: Norma E.030 2003, 2016 y 2018

\section{METODOLOGÍA}

Los modelos presentados son estructuras de 8 niveles destinándose para uso de oficinas de computación conformados por el sistema de pórticos con elementos estructurales de concreto armado, se caracterizan por ser uno de los sistemas más construidos en la ciudad de Lima y 
sus características geométricas son el resultado de evaluar diferentes planos, con el interés de forzar criterios de irregularidad torsional. Para lograr los objetivos del estudio se presenta un diagrama de flujo de los procesos más importantes partiendo desde los datos y estructuración, predimensionado, estudios de las tres normativas, desarrollo del modelo en el programa computacional Etabs 17, condiciones del análisis estructural, análisis comparativo y finalmente el diseño estructural como se muestra en la Figura 1.

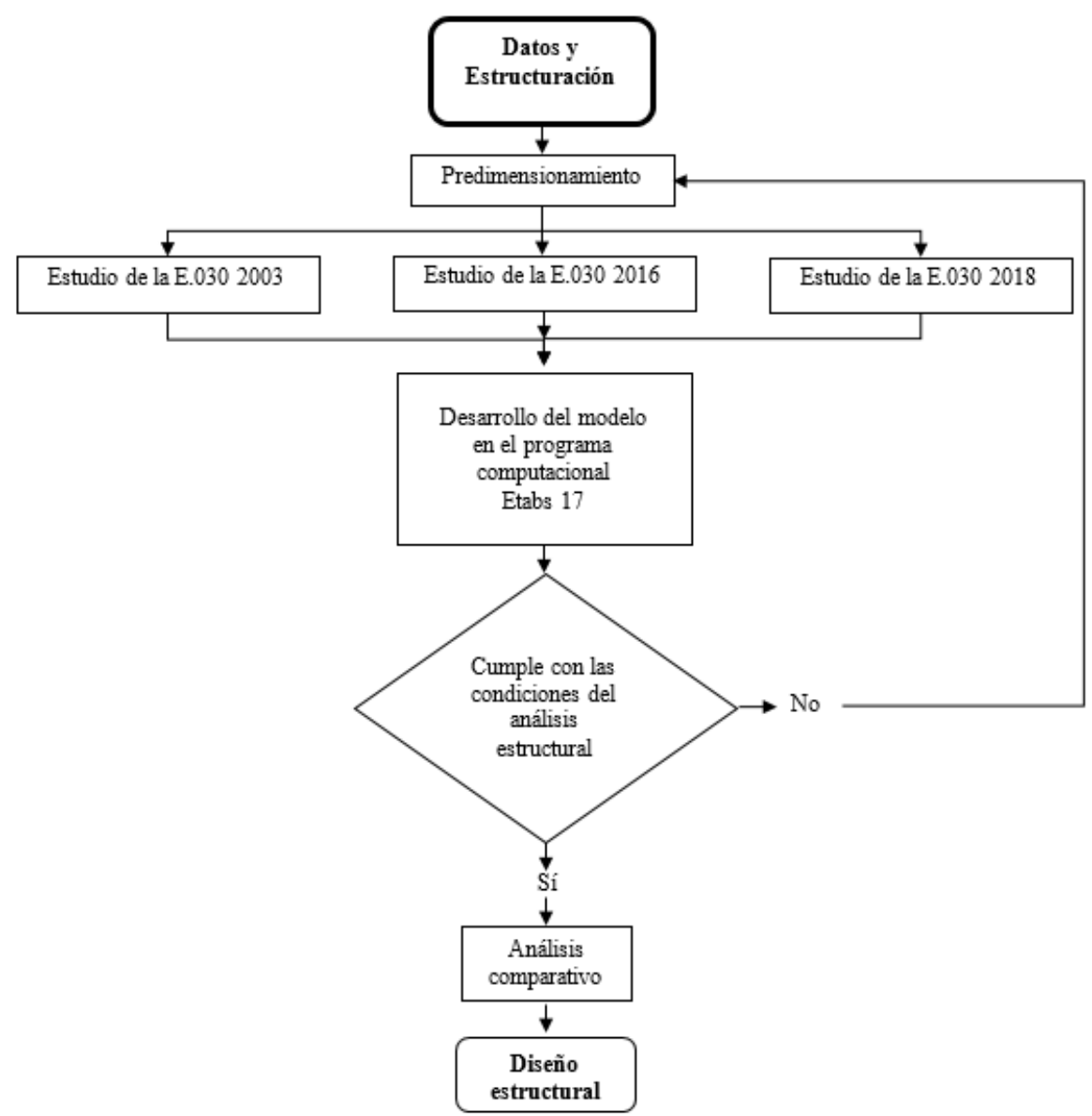

Figura. 1. Diagrama de flujo de la metodología para el diseño estructural. Fuente: los autores

\subsection{Datos y Estructuración}

La investigación presenta dos estructuras de concreto armado con sistema de pórticos de 8 niveles que se proyecta en la ciudad de Lima, por ser una zona con alto grado de peligro sísmico y uso de oficinas de computación. La estructuración está dada por losas, vigas, columnas, la cimentación está apoyada sobre un suelo rígido $S 1$ grava arenosa medianamente densa GP según la zonificación sísmica del Centro Peruano-Japonés de Investigaciones Sísmicas y Mitigación de Desastres CISMID [11]. Así mismo los parámetros geotécnicos se consideraron de acuerdo a los antecedentes e investigaciones realizadas en Lima para el análisis comparativo con una capacidad portante admisible qa promedio de $40 \mathrm{MPa}$ por medio 
ANÁLISIS COMPARATIVO DEL DISEÑO ESTRUCTURAL DE UNA EDIFICACIÓN REGULAR E IRREGULAR DE OCHO NIVELES EN SISTEMA DE PÓRTICOS APLICANDO LA NORMA E.030 2003, 2016 Y 2018 DISEÑO SISMORRESISTENTE EN LA CIUDAD DE LIMA

de zapatas aisladas centradas, desplantadas a una profundidad mínima Df de 1,20 $\mathrm{m}$ y el valor del módulo de reacción del suelo (Balasto/ Winkler) en función qa de 8000 MPa [12].

\subsection{Predimensionamiento}

La sobre carga para el uso de oficinas de computación es de s/c=250 kg/m y tabiquería de 76 $\mathrm{kg} / \mathrm{m}^{2}$ considerando el área de techado, volúmenes y peso específico de las mismas, además las propiedades de los materiales utilizados son: peso específico del concreto $\gamma=2400 \mathrm{~kg} / \mathrm{m}^{3}$, módulo de elasticidad del concreto $\mathrm{E}=15000 \times 10000 \times \sqrt{\mathrm{f}^{\prime} \mathrm{c}} \mathrm{kg} / \mathrm{m}^{2}$, módulo de Poisson del concreto $u=0,2$, resistencia a la compresión del concreto $f_{C^{\prime}}=2800000 \mathrm{~kg} / \mathrm{m}^{2}$, esfuerzo de fluencia del acero $f y^{\prime}=4,2 \times 10^{4} \mathrm{MPa}$. Los espacios entre ejes son de $6,00 \mathrm{~m}$ para ambas estructuras como se muestra en la Figura 2 y el dimensionamiento de los elementos estructurales basados en la norma de concreto armado [13], siendo las secciones iguales para ambas estructuras y en todos los niveles para realizar la comparación del comportamiento sísmico de acuerdo a las normativas E.030 diseño sismorresistente. La altura por nivel de $3,00 \mathrm{~m}$ y las secciones típicas de las columnas son de $80 \mathrm{~cm} \times 80 \mathrm{~cm}$, las vigas $40 \mathrm{~cm} \times 60 \mathrm{~cm}$, losas aligeradas con espesor de $30 \mathrm{~cm}$ para todos los niveles y la cimentación se modelo como fija empotrada considerando también la norma suelos y cimentaciones [14].

a)

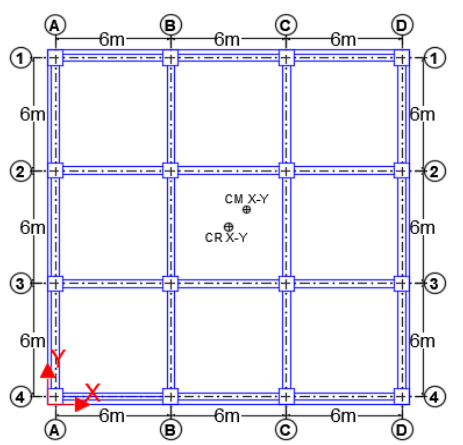

c)

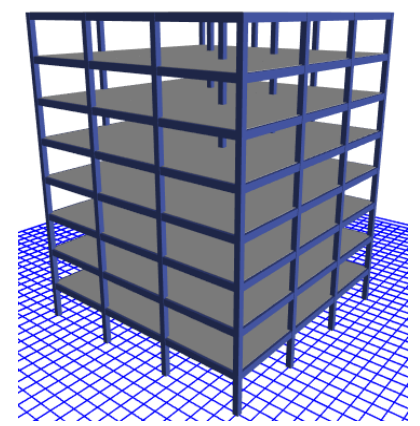

b)

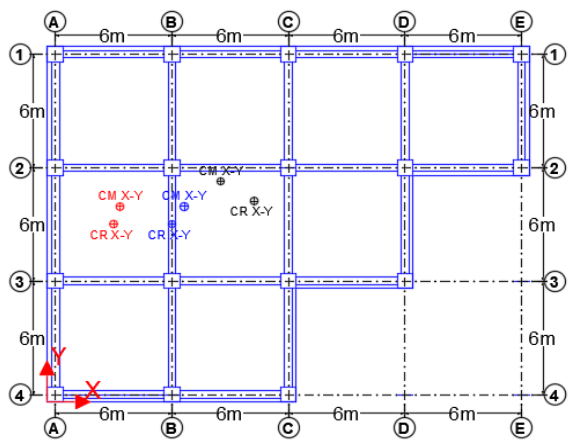

d)

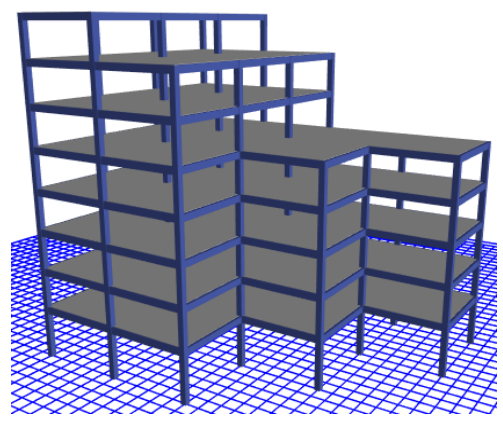

Figura 2. Plantas de: a) estructura regular, b) estructura irregular. Modelación de en 3D:c) estructura regular, d) estructura irregular. Fuente: los autores 


\subsection{Estudios de las Normativas E.030 2003, 2016 y 2018}

El estudio de la norma diseño sismorresistente E.030 se da para conocer los parámetros que influyen directamente al diseño. La principal causa de falla en los pórticos son los efectos torsionales, inducidas por las irregularidades y características de las estructuras detallándose los parámetros y sus modificaciones en el capítulo del desarrollo y resultado como el factor de zona $Z$, importancia $U$, amplificación sísmica $C$, suelo $S$, coeficiente de reducción $R$ y el método de análisis [15].

\subsection{Desarrollo del Modelo en el Programa Computacional Etabs 17}

En la investigación se utilizó modelos en 3D mediante el programa Etabs 17, siendo un software innovador y revolucionario evaluando la normativa E.030 [16]. Las estructuras fueron modeladas según el análisis estático y dinámico espectral estudiando las cortantes en la base, distribución de fuerzas sísmicas en altura, periodos de vibración, número de modos, porcentaje de masa participativa, espectro de diseño, criterios de combinación cuadrática completa $C Q C$ y finalmente controlando la distorsión de entrepiso considerando la distorsión límite.

\subsection{Análisis Comparativo}

Para el análisis comparativo se establecieron variables utilizando los parámetros de la norma E.030 diseño sismorresistente descritas anteriormente, de acuerdo a los siguientes parámetros: periodos de vibración, porcentaje de masa participativa, espectro de diseño, distorsión de entrepiso, fuerza cortante basal estática, fuerza cortante basal dinámica y las fuerzas máximas de diseño (momento flector, cortante y axial).

\subsection{Diseño Estructural}

El diseño de cada elemento estructural se realizó en base a los resultados del análisis comparativo, encontrando las diferencias entre las normas E.030 2003, 2016 y 2018 diseño sismorresitente para ambas estructuras. Para los cálculos se utilizaron fundamentos de diseño en concreto armado según la E.060 y ACI 318-14 asegurando que las estructuras tengan un buen comportamiento dúctil, siendo capaz de sufrir grandes deformaciones bajo cargas de servicio considerando los requisitos, factores, verificaciones, estados límites y cuantías de refuerzo para el diseño de losas aligeradas, columnas y vigas mediante el programa Etabs 17. Las cimentaciones con la norma E.050 [14] y ayuda del programa SAFE, donde se controlaron las condiciones de resistencia qa y asentamientos tolerables y diferenciales de las estructuras.

Revista Gaceta Técnica. Artículo de Investigación. 23(1), 48-71, enero-julio, 2022 
ANÁLISIS COMPARATIVO DEL DISEÑO ESTRUCTURAL DE UNA EDIFICACIÓN REGULAR E IRREGULAR DE OCHO NIVELES EN SISTEMA DE PÓRTICOS APLICANDO LA NORMA E.030 2003, 2016 Y 2018 DISEÑO SISMORRESISTENTE EN LA CIUDAD DE LIMA

\section{ANÁLISIS DE RESULTADOS}

\subsection{Centro de Masas y Rigidez}

En la Tabla 6 y la Figura 2 mostrada anteriormente, se puede observar el centro de masa $C M$ para ambas estructuras considerando una excentricidad del 5\%, siendo el lugar geométrico donde se genera el movimiento y el centro de rigidez $C R$ lugar geométrico donde la estructura se deforma menos.

Tabla 6. Centro de masa y centro de rigidez. Fuente: los autores

\begin{tabular}{|c|c|c|c|c|c|c|c|c|}
\hline \multirow[b]{2}{*}{ Nivel } & \multicolumn{5}{|c|}{ Estructura regular } & \multicolumn{3}{|c|}{ Estructura irregular } \\
\hline & $\begin{array}{c}\mathbf{C M} \\
\mathbf{X}-\mathbf{X}(\mathbf{m})\end{array}$ & $\begin{array}{c}\text { CM } \\
\mathbf{Y}-\mathbf{Y}(\mathbf{m})\end{array}$ & $\begin{array}{c}\text { CR } \\
\mathbf{X}-\mathbf{X}(\mathbf{m})\end{array}$ & $\begin{array}{c}\text { CR } \\
Y-Y(m)\end{array}$ & $\begin{array}{c}\text { CM } \\
\mathbf{X - X}(\mathbf{m})\end{array}$ & $\begin{array}{c}\mathbf{C M} \\
\mathrm{Y}-\mathrm{Y}(\mathbf{m})\end{array}$ & $\begin{array}{c}\text { CR } \\
X-X(m)\end{array}$ & $\begin{array}{c}\text { CR } \\
\mathrm{Y}-\mathrm{Y}(\mathrm{m})\end{array}$ \\
\hline 8 & 10,34 & 10,34 & 9,4 & 9,4 & 3,74 & 10,34 & 3,4 & 9,4 \\
\hline 7 & 10,34 & 10,34 & 9,4 & 9,4 & 7,04 & 10,34 & 6,4 & 9,4 \\
\hline 6 & 10,34 & 10,34 & 9,4 & 9,4 & 7,04 & 10,34 & 6,4 & 9,4 \\
\hline 5 & 10,34 & 10,34 & 9,4 & 9,4 & 8,92 & 11,66 & 10,63 & 10,63 \\
\hline 4 & 10,34 & 10,34 & 9,4 & 9,4 & 8,92 & 11,66 & 10,63 & 10,63 \\
\hline 3 & 10,34 & 10,34 & 9,4 & 9,4 & 8,92 & 11,66 & 10,63 & 10,63 \\
\hline 2 & 10,34 & 10,34 & 9,4 & 9,4 & 8,92 & 11,66 & 10,63 & 10,63 \\
\hline 1 & 10,34 & 10,34 & 9,4 & 9,4 & 8,92 & 11,66 & 10,63 & 10,63 \\
\hline
\end{tabular}

\subsection{Pesos Sísmicos}

Las sobrecargas para el peso sísmico se utilizan las mencionadas en el predimensionado, considerando el $100 \%$ de la carga muerta $C M$ y $25 \%$ de la carga viva $C V$ por ser de categoría $C$ de acuerdo al uso de la estructura según indica la E.030. Asimismo, la gravedad $g=$ $9,8066 \mathrm{~m} / \mathrm{s}^{2}$ para las masas traslacionales " $X$ " e " $Y$ " y considerando las inercias y sus respectivas áreas para la masa rotacional en $Z$ como se muestra en la Tabla 7.

Tabla 7. Pesos sísmicos de las estructuras. Fuente: los autores

\begin{tabular}{|c|c|c|c|c|c|c|}
\hline \multicolumn{7}{|c|}{ Peso de las estructuras $100 \% \mathrm{CM}+25 \% \mathrm{CV}$} \\
\hline & \multicolumn{3}{|c|}{ Estructura regular } & \multicolumn{3}{|c|}{ Estructura irregular } \\
\hline Nivel & Peso (ton) & $\begin{array}{c}\text { Masa X e Y } \\
\left(\text { ton.s }{ }^{2} / \mathrm{m}\right)\end{array}$ & $\begin{array}{c}\text { Masa Z } \\
\left(\text { ton.s }{ }^{2} . m\right)\end{array}$ & Peso (ton) & $\begin{array}{c}\text { Masa X e Y } \\
\left(\text { ton.s }{ }^{2} / m\right)\end{array}$ & $\begin{array}{c}\text { Masa Z } \\
\left(\text { ton.s }^{2} \mathrm{~m}\right)\end{array}$ \\
\hline 8 & 277,626 & 28,310 & 1667,649 & 112,818 & 11,504 & 383,169 \\
\hline 7 & 312,970 & 31,914 & 1879,953 & 219,286 & 22,361 & 963,908 \\
\hline 6 & 312,970 & 31,914 & 1879,953 & 219,286 & 22,361 & 963,908 \\
\hline 5 & 312,970 & 31,914 & 1879,953 & 322,047 & 32,840 & 2276,559 \\
\hline 4 & 312,970 & 31,914 & 1879,953 & 322,047 & 32,840 & 2276,559 \\
\hline 3 & 312,970 & 31,914 & 1879,953 & 322,047 & 32,840 & 2276,559 \\
\hline 2 & 312,970 & 31,914 & 1879,953 & 322,047 & 32,840 & 2276,559 \\
\hline 1 & 337,546 & 34,420 & 2027,577 & 348,159 & 35,502 & 2461,146 \\
\hline TOTAI & 2492,995 & 254,215 & 14974,944 & 2187,739 & 223,087 & 13878,367 \\
\hline
\end{tabular}

\subsection{Periodo Fundamental $T$}

En la Tabla 8 se muestra en ambas direcciones la estructura regular con periodo fundamental de $T x=0,728 \mathrm{~s}$ y $T y=0,728 \mathrm{~s}$ y la estructura irregular con periodo fundamental de $T x=0,619 \mathrm{~s}$ 
y $T y=0,664 \mathrm{~s}$. El periodo dependerá directamente de la rigidez de las estructuras y por consecuentes se refleja en sus modos de vibración.

Tabla 8. Periodos y masas participativas. Fuente: los autores

\begin{tabular}{|c|c|c|c|c|c|c|}
\hline \multirow[b]{2}{*}{ Modo } & \multicolumn{3}{|c|}{ Estructura regular } & \multicolumn{3}{|c|}{ Estructura irregular } \\
\hline & Periodo (s) & $\begin{array}{c}\text { Masa } \\
\text { Participativa } \\
\text { Acumulada } \\
\text { X-X }(\%)\end{array}$ & $\begin{array}{c}\text { Masa } \\
\text { Participativa } \\
\text { Acumulada } \\
\text { Y-Y }(\%)\end{array}$ & Periodo (s) & $\begin{array}{c}\text { Masa } \\
\text { Participativa } \\
\text { Acumulada } \\
\text { X-X }(\%)\end{array}$ & $\begin{array}{c}\text { Masa } \\
\text { Participativa } \\
\text { Acumulada } \\
\text { Y-Y }(\%)\end{array}$ \\
\hline 1 & 0,744 & 39,01 & 39,01 & 0,664 & 0,03 & 73,23 \\
\hline 2 & 0,728 & 80,17 & 80,17 & 0,619 & 80,94 & 73,26 \\
\hline 3 & 0,553 & 82,32 & 82,32 & 0,446 & 80,94 & 81,63 \\
\hline 4 & 0,232 & 87,30 & 87,30 & 0,222 & 88,32 & 84,14 \\
\hline 5 & 0,227 & 92,57 & 92,57 & 0,216 & 91,13 & 91,28 \\
\hline 6 & 0,173 & 92,86 & 92,86 & 0,165 & 91,36 & 91,81 \\
\hline
\end{tabular}

\subsection{Comparación de Periodos de la E.030 2003, 2016 y 2018}

En la Tabla 9 se observa que los periodos de la norma sismorresistente tiene una variación máxima de 17,61\% para la estructura regular comparando el periodo modal con el alternativo, y para la estructura irregular una variación máxima en la dirección $X-X$ y $Y-Y$ de 30,42\% y $27,27 \%$ respectivamente siendo la comparación del periodo normativo con el alternativo quien da mayores valores del factor de amplificación sísmica $C$, se optó por trabajar con los periodos normativos y modales al ser las más utilizadas en diseño sísmico.

Tabla 9. Variación de periodos. Fuente: los autores

\begin{tabular}{ccccc}
\hline \multirow{2}{*}{ Periodos (s) } & \multicolumn{2}{c}{ Estructura regular } & \multicolumn{2}{c}{ Estructura irregular } \\
\cline { 2 - 5 } & $\begin{array}{c}\text { E.030 2003, } \\
\mathbf{2 0 1 6} \text { y 2018 }\end{array}$ & $\begin{array}{c}\text { Variación } \\
(\mathbf{\%})\end{array}$ & $\begin{array}{c}\text { E.030 2003, } \\
\mathbf{2 0 1 6} \mathbf{~ 2 0 1 8}\end{array}$ & $\begin{array}{c}\text { Variación } \\
(\mathbf{\%})\end{array}$ \\
\hline Tx Normativo & 0,686 & 6,12 & 0,686 & 10,82 \\
Tx Modal & 0,728 & 17,61 & 0,619 & 17,68 \\
Tx Alternativ) & 0,619 & 10,82 & 0,526 & 30,42 \\
Ty Normativo & 0,686 & 6,12 & 0,686 & 3,31 \\
Ty Modal & 0,728 & 17,61 & 0,664 & 23,19 \\
Ty Alternativo & 0,619 & 10,82 & 0,539 & 27,27 \\
\hline
\end{tabular}

\subsection{Estructura Regular}

\section{Análisis estático}

La expresión de la cortante estática es la resistencia que se debe dar a una estructura para que tenga un buen comportamiento ante los terremotos, lográndose entender que no colapsen en sismos de gran magnitud y no se agrieten demasiado en los de menos magnitud. La ingeniería sigue en constante estudio de cada parámetro sísmico normativo, haciendo teorías que se van mejorando con el tiempo y se van adaptando a la realidad. En la Tabla 10 se observa los 
ANÁLISIS COMPARATIVO DEL DISEÑO ESTRUCTURAL DE UNA EDIFICACIÓN REGULAR E IRREGULAR DE OCHO NIVELES EN SISTEMA DE PÓRTICOS APLICANDO LA NORMA E.030 2003, 2016 Y 2018 DISEÑO SISMORRESISTENTE EN LA CIUDAD DE LIMA

parámetros sísmicos para estructuras proyectadas en la ciudad de Lima con tipo de suelo rígido $S 1$ y uso común $U$, factor de amplificación sísmica $C$, coeficiente de reducción $R$ para pórticos y el cambio en la zonificación $Z$ por la actualización de la norma del 2003 al 2016 2018 presentando una variación máxima de $6,17 \%$ de las cortantes estáticas entre el periodo normativo y periodo modal para cada normativa E.030. Así mismo, una variación del 12,50\% en las cortantes utilizando periodos normativos o modal según la norma del 2003 en comparación del 2016-2018.

Tabla 10.Variación de la cortante estática. Fuente: los autores

\begin{tabular}{|c|c|c|c|c|c|c|}
\hline & \multicolumn{2}{|c|}{ E.030 2003} & \multicolumn{2}{|c|}{ E.030 2016} & \multicolumn{2}{|c|}{ E.030 2018} \\
\hline & $\begin{array}{c}T \\
\text { Norma }\end{array}$ & $\begin{array}{c}T \\
\text { Modal }\end{array}$ & $\begin{array}{c}T \\
\text { Norma }\end{array}$ & $\begin{array}{c}T \\
\text { Modal }\end{array}$ & $\begin{array}{c}T \\
\text { Norma }\end{array}$ & $\begin{array}{c}T \\
\text { Modal }\end{array}$ \\
\hline & X-Y & $\mathrm{X}-\mathrm{Y}$ & X-Y & X-Y & X-Y & X-Y \\
\hline$Z$ & 0,4 & 0,4 & 0,45 & 0,45 & 0,45 & 0,45 \\
\hline$U$ & 1,00 & 1,00 & 1,00 & 1,00 & 1,00 & 1,00 \\
\hline$C$ & 1,45 & 1,37 & 1,45 & 1,37 & 1,45 & 1,37 \\
\hline$S$ & 1,00 & 1,00 & 1,00 & 1,00 & 1,00 & 1,00 \\
\hline$R$ & 8,00 & 8,00 & 8,00 & 8,00 & 8,00 & 8,00 \\
\hline$Z U C S / R$ & 0,072 & 0,068 & 0,082 & 0,077 & 0,082 & 0,077 \\
\hline Peso(ton) & 2492,99 & 2492,99 & 2492,99 & 2492,99 & 2492,99 & 2492,99 \\
\hline V Estática(ton) & 181,78 & 171,22 & 204,50 & 192,62 & 204,50 & 192,62 \\
\hline Variación* (\%) & \multicolumn{2}{|c|}{6,17} & \multicolumn{2}{|c|}{6,17} & \multicolumn{2}{|c|}{6,17} \\
\hline Variación** (\%) & \multicolumn{6}{|c|}{12,50} \\
\hline
\end{tabular}

Las fuerzas laterales se distribuyen para todos los pisos como se muestra en la Figura 3, algunas dependen de periodos $T$ normativos y modales. De acuerdo al periodo normativo existe un incremento de $17,16 \%$ en el último nivel que decrece ligeramente hasta el primer nivel, y de acuerdo al periodo $T$ modal existe descenso de $-1,62 \%$ para el ultimo nivel y un incremento de $22,68 \%$ en el penúltimo nivel que decrece ligeramente hasta el primer nivel, según la norma del 2003 en comparación del 2016-2018.

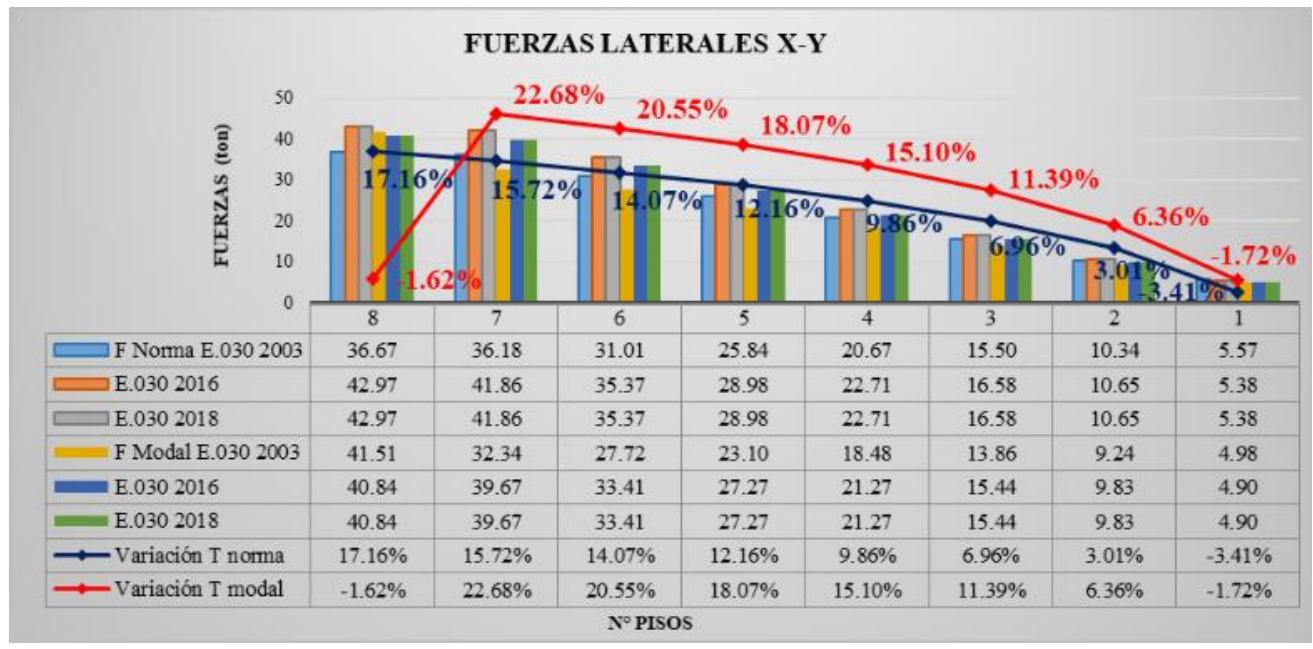

Figura 3. Variación de las fuerzas laterales en X-Y. Fuente: los autores 
Las derivas inelásticas para estructuras regulares no tienen modificación desde la norma del 2003, por lo que los desplazamientos laterales se mantienen multiplicándose por $0,75 R$ los resultados obtenidos del análisis lineal y elástico con las solicitaciones reducidas. Logrando ver en la Figura 4, las derivas máximas inelásticas en ambas direcciones de acuerdo a fuerzas que dependen de periodos $T$ normativos y modales, se determina que el incremento de las fuerzas laterales para la norma del 2016 - 2018 afectan directamente a la deriva y según el periodo $T$ normativo, mientras la norma del 2003 presenta una distorsión máxima en el tercer nivel de 0,00515 para la norma del 2016 y 2018 con una distorsión máxima de 0,00585 llegando a tener una variación de $13,59 \%$ y según el periodo $T$ modal

Por otro lado, la norma del 2003 presentaba una distorsión máxima en el tercer nivel de 0,00488, para la norma del 2016 y 2018 la distorsión máxima es de 0,00552 llegando a tener una variación de $13,12 \%$. También se logra cumplir con la deriva máxima de 0,007 que permite la norma peruana E.030.

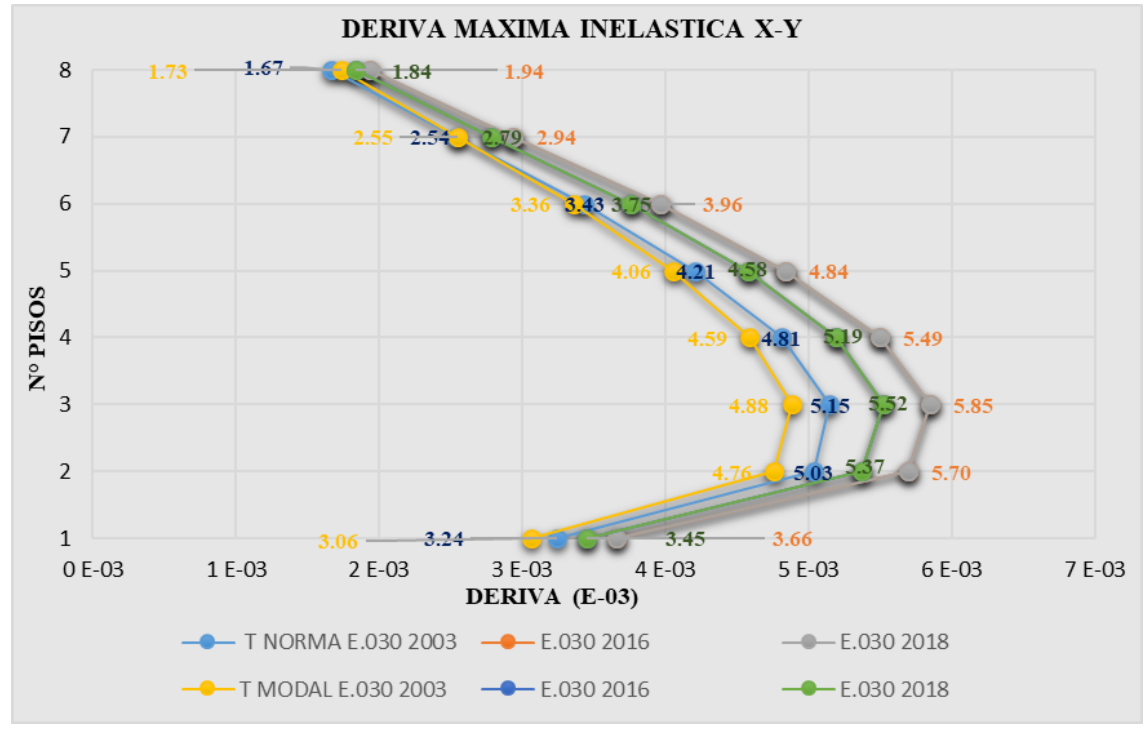

Figura 4. Variación de las derivas máximas inelásticas $X-Y$. Fuente: los autores Análisis dinámico

En la Figura 5 se presentan los dos espectros de diseño por que la norma del 2016 y 2018 son iguales en ambas direcciones, presentando las modificaciones de las normas en donde se incrementó las pseudo aceleraciones de 1,23 a 1,38 m/s² de la norma 2003 a 2016-2018 respectivamente y en las normas del 2016 y 2018 se modifica la curvatura del último sector para periodos mayores a $T L$, siendo estas aceleraciones menores en comparación con la norma del 2003.

Mientras que en la Figura 6, las derivas máximas inelásticas en ambas direcciones se incrementaron por los cambios de los espectros de diseño del 2016 y 2018. Según la norma 
ANÁLISIS COMPARATIVO DEL DISEÑO ESTRUCTURAL DE UNA EDIFICACIÓN REGULAR E IRREGULAR DE OCHO NIVELES EN SISTEMA DE PÓRTICOS APLICANDO LA NORMA E.030 2003, 2016 Y 2018 DISEÑO SISMORRESISTENTE EN LA CIUDAD DE LIMA

del 2003, se tiene una distorsión máxima en el tercer nivel de 0,0038 y para la norma del 2016 y 2018 presenta una distorsión máxima de 0,0043 llegando a tener una variación de 13,16\%.

Pero se logra cumplir con la deriva máxima de 0,007 que permite la norma peruana E.030.

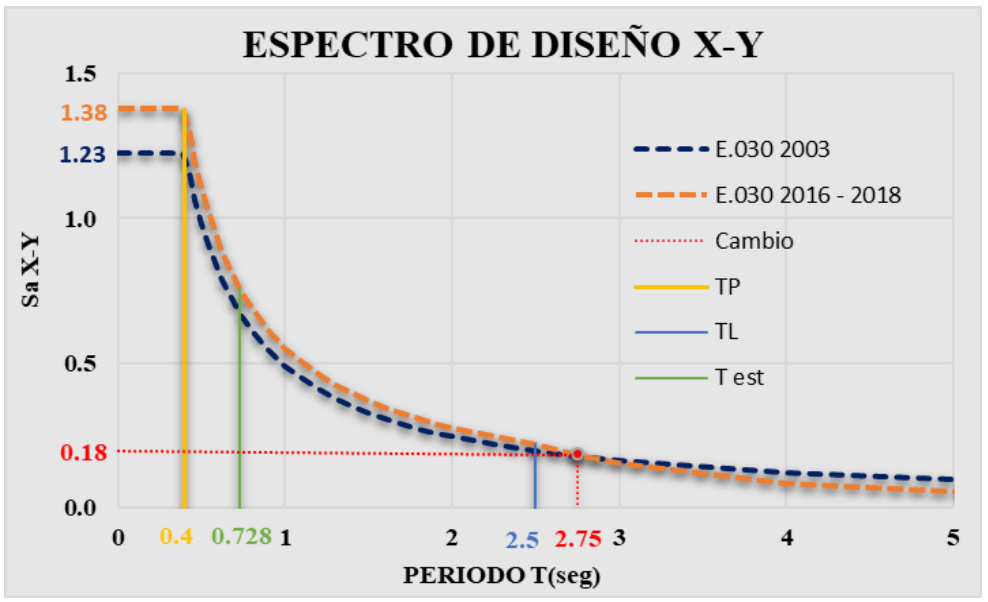

Figura 5. Variación de espectros de diseño $X-Y$. Fuente: los autores

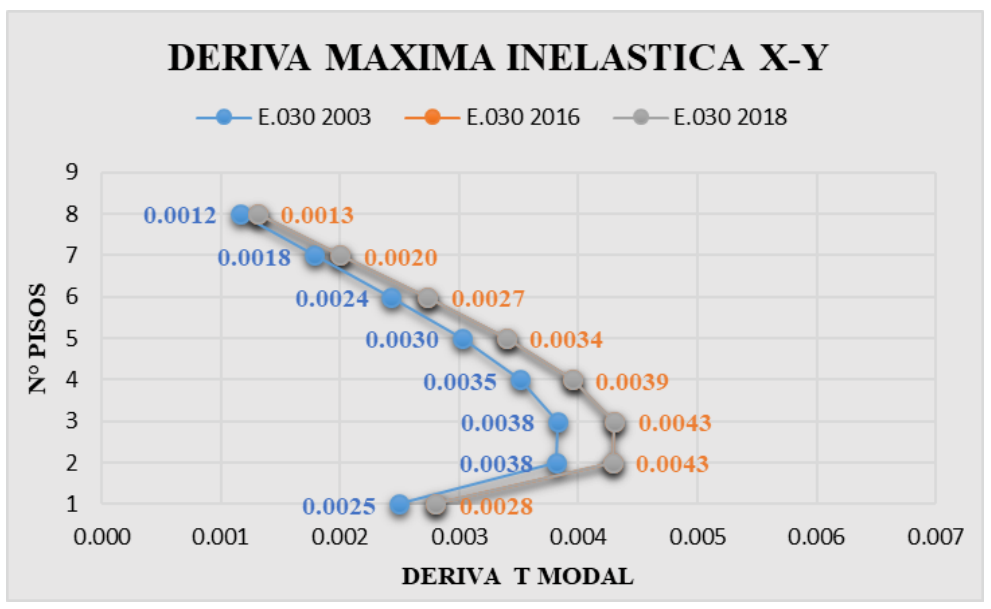

Figura 6. Variación de la deriva máxima inelástica X-Y. Fuente: los autores

La norma E.030 del 2003, 2016 y 2018 considera que la fuerza cortante basal en el primer entrepiso no puede ser menor que el $80 \%$ del valor calculado según el análisis estático. En la Tabla 11 se muestran las cortantes estáticas y dinámicas según periodos $T$ normativos y modales obteniendo un factor de escala $\left(0,8 V_{\text {estática }} / V_{\text {dinámica }}\right)$ de 1,0401 y 1 para poder cumplir con la cortante mínima y obtener finalmente una cortante basal corregida $V$ basal.

Tabla 11. Cortante basal corregida. Fuente: los autores

\begin{tabular}{cccccc}
\hline Norma & Dirección & V estática (ton) & V dinámica (ton) & Factor Esc. (F) & V basal (ton) \\
\hline \multirow{2}{*}{ E.030 2003 } & T Norma X-Y & 181,7809 & 140,6888 & 1,0401 & 146,3304 \\
& T Modal X-Y & 171,2222 & 140,6888 & 1 & 140,6888 \\
E.030 2016 & T Norma X-Y & 204,5035 & 158,28 & 1,0401 & 164,6270 \\
& T Modal X-Y & 192,6250 & 158,28 & 1 & 158,28 \\
\multirow{2}{*}{ E.030 2018 } & T Norma X-Y & 204,5035 & 158,28 & 1,0401 & 164,6270 \\
& T Modal X-Y & 192,6250 & 158,28 & 1 & 158,28 \\
\hline
\end{tabular}

Las fuerzas máximas de diseño surgen en las columnas del primer piso, considerando que Revista Gaceta Técnica. Artículo de Investigación. 23(1), 48-71, enero-julio, 2022 
ambas direcciones son iguales por ser una estructura regular. Las fuerzas dependerán del análisis estático, dinámico, periodo $T$ normativa, modal y las actualizaciones de las normas E.030 2003, 2016 y 2018. Las fuerzas máximas son de los pórticos (2-2) y (C-C) en la dirección $X X-Y Y$ como se muestra en la Figura 7, según las fuerzas estáticas del periodo $T$ modal comparando la norma del 2003 con la 2016 y 2018 se tiene una variación de 11,96\%, $12,92 \%$ y $12,62 \%$ de fuerzas axiales, cortante y momentos flectores respectivamente y para las fuerzas dinámicas con un $12,5 \%$ en fuerzas axiales, cortantes y momentos flectores y aun así ningún pórtico supera el $30 \%$ de cortante basal por lo que no presenta redundancia estructural y cumple con las distorsiones calculadas anteriormente. Se observa la modelación de la estructura regular en la Figura 8.

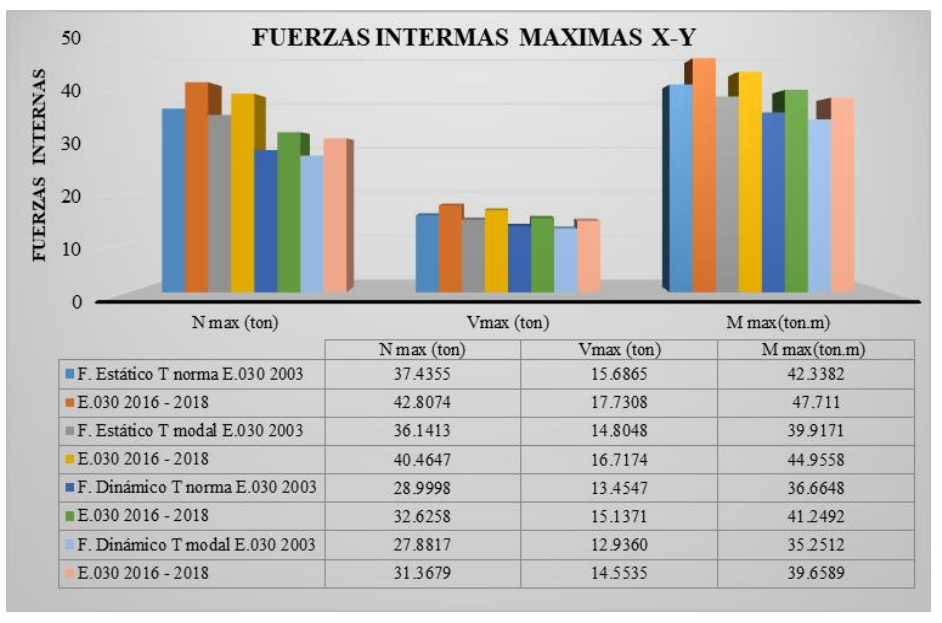

Figura 7. Fuerzas internas de diseño. Fuente: los autores

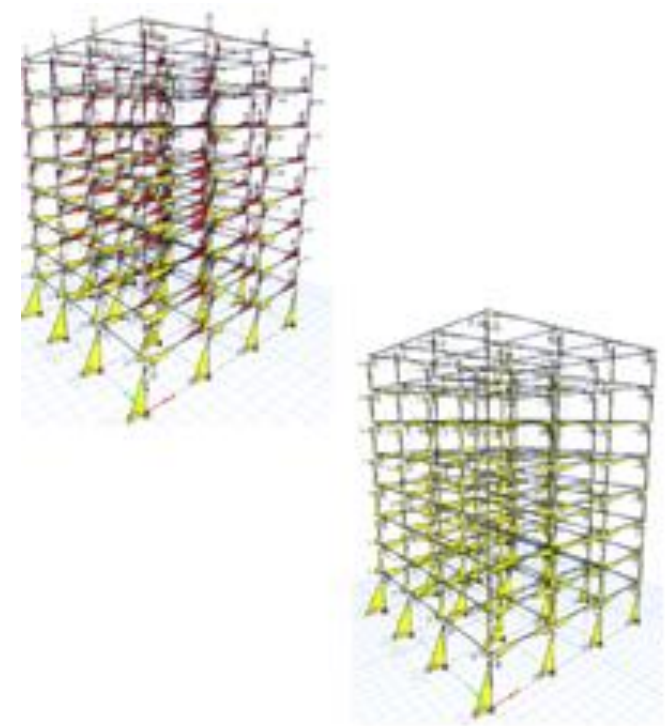

Figura 8. Modelación de la estructura regular. Fuente: los autores 
ANÁLISIS COMPARATIVO DEL DISEÑO ESTRUCTURAL DE UNA EDIFICACIÓN REGULAR E IRREGULAR DE OCHO NIVELES EN SISTEMA DE PÓRTICOS APLICANDO LA NORMA E.030 2003, 2016 Y 2018 DISEÑO SISMORRESISTENTE EN LA CIUDAD DE LIMA

\subsection{Estructura Irregular}

\section{Análisis estático}

- La norma del 2003 considera $3 / 4$ del coeficiente $R$ si presenta irregularidad: la estructura tiene irregularidad en altura de geometría vertical y en planta esquina entrante calculada según la Tabla 3. Por ello se considera 0,75 en Ip para ambas direcciones.

- La norma del 2016 considera factores de acuerdo al tipo de irregularidad que se presente en cada dirección: la estructura presenta irregularidad en altura geometría vertical $I a=0,9$ y rigidez - piso blando $I a=0,75$ en ambas direcciones, considerando irregularidad de rigidez - piso blando por ser el menor valor cuando la distorsión de entrepiso es mayor que 1,25 veces el promedio de las distorsiones de entrepiso en los tres niveles superiores adyacentes. También presenta irregularidad en planta de esquina entrante $I p=0,9$ en ambas direcciones y torsión $I p=0,75$ en la dirección $Y Y$, considerando irregularidad torsional por ser el menor valor cuando el desplazamiento máximo relativo de entrepiso en un extremo es mayor que 1,2 veces el desplazamiento relativo del centro de masa. Por ello según lo mencionado y calculado de acuerdo a la Tabla 3 se tiene un $I a=0,75$ e $I p=0,75$ para ambas direcciones.

- La norma del 2018 considera factores de acuerdo al tipo de irregularidad que se presenta en cada dirección: la estructura presenta irregularidad en altura geometría vertical $I a=0,9$ y también la presenta en planta de esquina entrante $I p=0,9$ en ambas direcciones y torsión $I p=0,75$ en la dirección $Y Y$, considerando irregularidad torsional por ser el menor valor cuando el desplazamiento máximo relativo de entrepiso en un extremo es mayor que 1,3 veces el desplazamiento relativo promedio de los extremos del mismo entrepiso. Por ello según lo mencionado y calculado de acuerdo a la Tabla 3 se tiene un $I a=0,9$ e $I p=$ 0,75 para ambas direcciones.

- Con variación máxima de 50\% de las cortantes estáticas entre la norma del 2003 y 2016, así mismo una variación de -16,67\% entre la norma del 2016 y 2018 para ambas direcciones como se muestra en la Tabla 12. 
Tabla 12. Variación de la cortante estática. Fuente: los autores

\begin{tabular}{ccccccc}
\hline & \multicolumn{2}{c}{ E.030 2003 } & \multicolumn{2}{c}{ E.030 2016 } & \multicolumn{2}{c}{ E.030 2018 } \\
\hline & $\mathbf{X}$ & $\mathbf{Y}$ & $\mathbf{X}$ & $\mathbf{Y}$ & $\mathbf{X}$ & $\mathbf{Y}$ \\
\hline$Z$ & 0,40 & 0,40 & 0,45 & 0,45 & 0,45 & 0,45 \\
$U$ & $\mathrm{c}$ & 1,00 & 1,00 & 1,00 & 1,00 & 1,00 \\
$C$ & 1,61 & 1,50 & 1,61 & 1,50 & 1,61 & 1,50 \\
$S$ & 1,00 & 1,00 & 1,00 & 1,00 & 1,00 & 1,00 \\
$I a$ & 1,00 & 1,00 & 0,75 & 0,75 & 0,90 & 0,90 \\
$I p$ & 0,75 & 0,75 & 0,75 & 0,75 & 0,75 & 0,75 \\
$R o$ & 8,00 & 8,00 & 8,00 & 8,00 & 8,00 & 8,00 \\
$R$ & 6,00 & 6,00 & 4,50 & 4,50 & 5,40 & 5,40 \\
$Z U C S / R$ & 0,10 & 0,10 & 0,16 & 0,15 & 0,13 & 0,12 \\
Peso(ton) & 2187,73 & 2187,73 & 2187,73 & 2187,73 & 2187,73 & 2187,73 \\
\hline V Estática(ton) & 235,62 & 219,65 & 353,43 & 329,47 \\
\hline Variación* (\%) & \multicolumn{7}{c}{50} \\
\hline Variación** (\%) & \multicolumn{7}{c}{$-16,67$} \\
\hline
\end{tabular}

Nota: (*=variación de E.030 2003 y 2016) y (**=variación de E.030 2016 y 2018).

\section{Análisis dinámico}

Se presentan los espectros de diseño en la Figura 9 de la norma 2003, 2016 y 2018 para ambas direcciones, presentando las modificaciones de las normas donde se incrementó las pseudo aceleraciones de 1,63 a $2,45 \mathrm{~m} / \mathrm{s}^{2}$ y luego se reduce a $2,04 \mathrm{~m} / \mathrm{s}^{2}$ de la norma 2003 , 2016 y 2018 respectivamente y en las normas del 2016 y 2018 se modifica la curvatura del último sector para periodos mayores a $T L$, siendo estas aceleraciones menores en comparación con la norma del 2003.

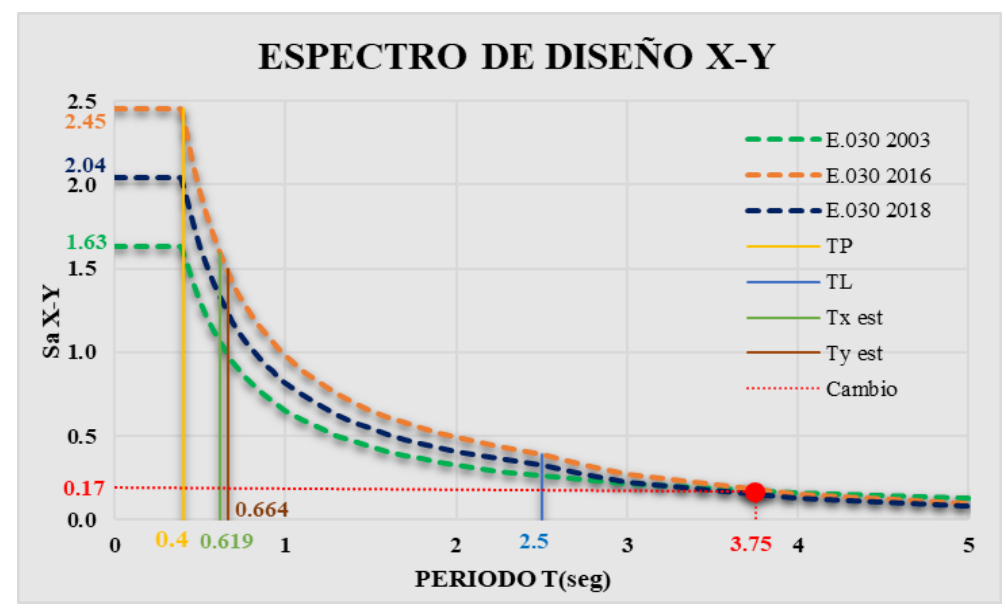

Figura 9. Variación de los espectros de diseño. Fuente: los autores

En la Figura 10, las derivas máximas inelásticas en ambas direcciones se modificaron por los cambios de los espectros de diseño. Para la dirección $X X$ según la norma del 2003 se tiene una distorsión máxima en el segundo nivel de 0,0036, incrementándose para la norma del 2016 con una distorsión máxima de 0,0054 con variación entre sí de 50\% y luego decrece para la norma del 2018 con distorsión máxima de 0,0046 y variación de -14,81\%. De la misma Revista Gaceta Técnica. Artículo de Investigación. 23(1), 48-71, enero-julio, 2022 
ANÁLISIS COMPARATIVO DEL DISEÑO ESTRUCTURAL DE UNA EDIFICACIÓN REGULAR E IRREGULAR DE OCHO NIVELES EN SISTEMA DE PÓRTICOS APLICANDO LA NORMA E.030 2003, 2016 Y 2018 DISEÑO SISMORRESISTENTE EN LA CIUDAD DE LIMA

manera para la dirección $Y Y$ según la norma del 2003 se tiene distorsión máxima en el segundo nivel de 0,0033, incrementándose para la norma del 2016 con distorsión máxima de 0,0049 con una variación entre sí de 48,48\% y luego decrece para la norma del 2018 con distorsión máxima de 0,0042 y variación de -14,29\%.
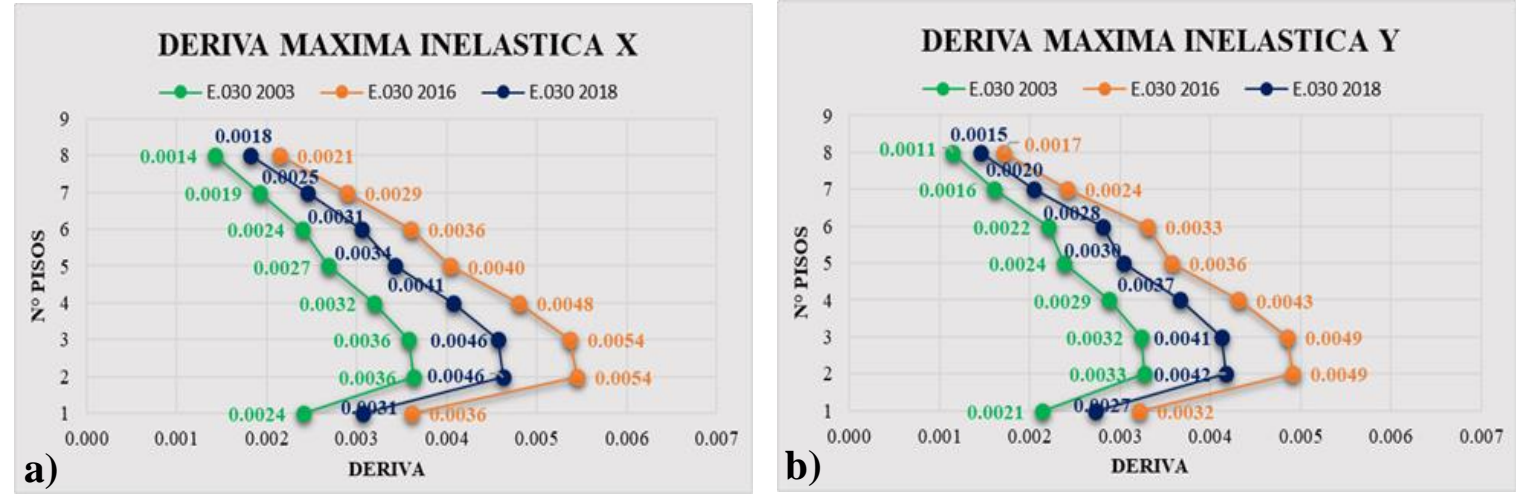

Figura 10. a) Variación de derivas $X-X$. b) Variación de derivas $Y-Y$. Fuente: los autores

La norma E.030 del 2003, 2016 y 2018 considera que la fuerza cortante basal en el primer entrepiso, no puede ser menor que el 90\% del valor calculado según el análisis estático. En la Tabla 13 se muestran las cortantes estáticas y dinámicas par ambas direcciones obteniendo un factor de escala $(0,9$ Vestática/ $V$ dinámica $)$ de 1,0762 y 1,1065 para poder cumplir con la cortante mínima y obtener finalmente una cortante basal corregida $V$ basal.

Tabla 13. Cortante basal corregida. Fuente: los autores

\begin{tabular}{cccccc}
\hline Norma & Dirección & $\boldsymbol{V}$ estática (ton) & $\boldsymbol{V}$ dinámica (ton) & Factor Esc. $(\mathbf{F})$ & $\boldsymbol{V}$ basal (ton) \\
\hline \multirow{2}{*}{ E.030 2003 } & $X$ & 235,6208 & 197,0464 & 1,0762 & 212,0613 \\
& $Y$ & 219,6525 & 178,6647 & 1,1065 & 197,6925 \\
\multirow{2}{*}{ E.030 2016 } & $X$ & 353,4312 & 295,5702 & 1,0762 & 318,0926 \\
& $Y$ & 329,4788 & 267,9954 & 1,1065 & 296,5369 \\
\multirow{2}{*}{ E.030 2018 } & $X$ & 294,5260 & 246,3086 & 1,0762 & 265,0773 \\
& $Y$ & 274,5657 & 223,335 & 1,1065 & 247,1202 \\
\hline
\end{tabular}

En la Figura 11 las fuerzas máximas de diseño surgen en las columnas del primer piso considerando que ambas direcciones son diferentes por ser una estructura irregular. Las fuerzas dependerán del análisis estático, dinámico, dirección y las actualizaciones de las normas E.030 2003, 2016 y 2018. Las fuerzas máximas son de los pórticos (2-2) y (A-A) en la dirección $X X-Y Y$ respectivamente con una variación similar al de las cortantes estáticas en la Tabla 12 y considerando que dichos pórticos superan el 30\% de la cortante basal será 1,25 veces su valor para ambas direcciones. Además, según las fuerzas estáticas y dinámicas en la dirección $Y-Y$ de la norma 2018 tiene una variación de 13,53\%, 5,65\% y 4,55\% de fuerzas axiales, cortante y momentos flectores respectivamente. Se observa la modelación de la estructura regular en la Figura 12. 


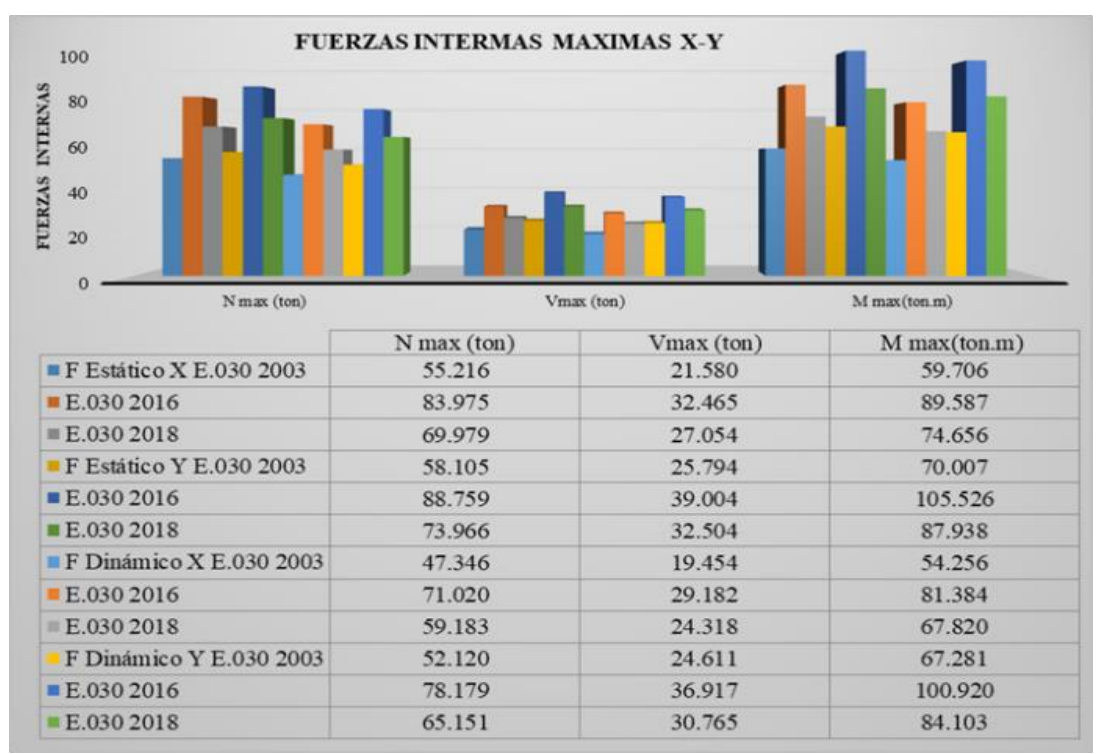

Figura 11. Fuerzas internas de diseño. Fuente: los autores

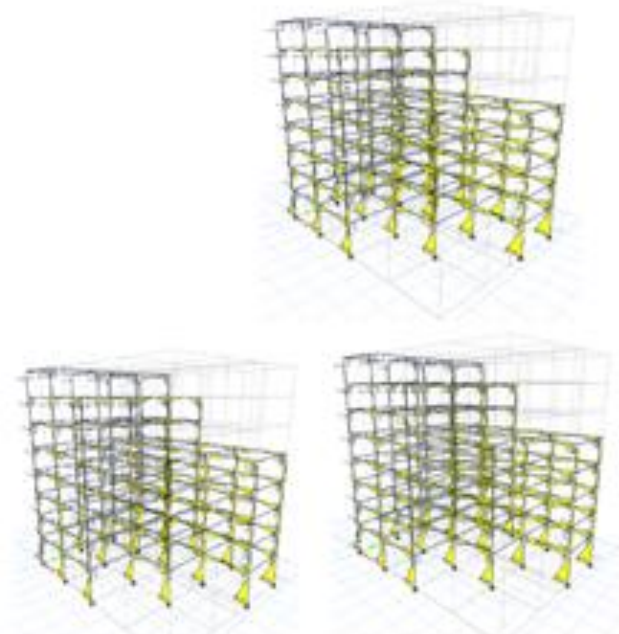

Figura 12. Modelación de la estructura irregular. Fuente: los autores

\subsection{Diseño Estructural}

\section{Losa aligerada}

La losa aligerada en una dirección no tiene modificación, ya que se diseñan por cargas de gravedad transmitiéndolas a las vigas, además, sirven como diafragma rígido para cada nivel y detallando las dimensiones en la Figura 13.

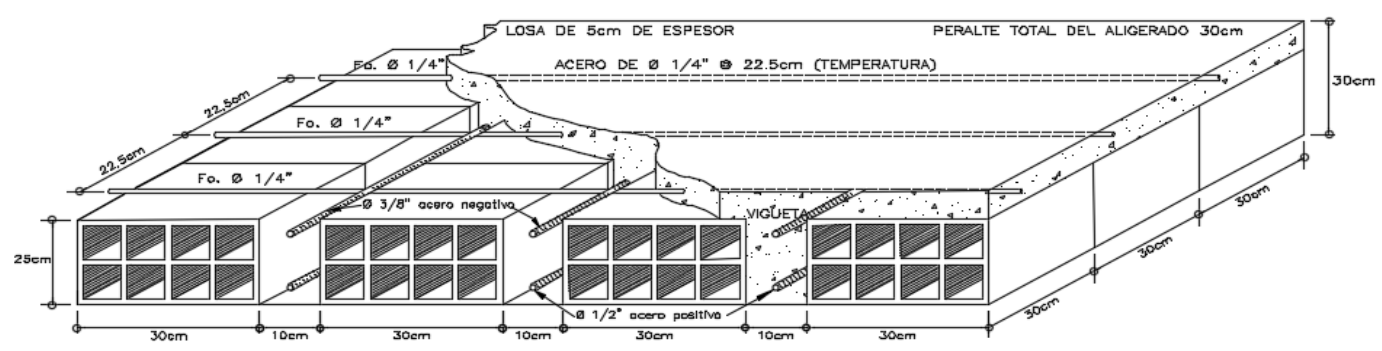

Figura 13. Detalles de la losa aligerada en una dirección. Fuente: los autores

Revista Gaceta Técnica. Artículo de Investigación. 23(1), 48-71, enero-julio, 2022 
ANÁLISIS COMPARATIVO DEL DISEÑO ESTRUCTURAL DE UNA EDIFICACIÓN REGULAR E IRREGULAR DE OCHO NIVELES EN SISTEMA DE PÓRTICOS APLICANDO LA NORMA E.030 2003, 2016 Y 2018 DISEÑO SISMORRESISTENTE EN LA CIUDAD DE LIMA

\section{Columnas}

En la Figura 14, las columnas no presentaron modificaciones por que se trabajó con una sección cuadrada de $80 \mathrm{~cm}$, la cual debía cumplir con las tres normativas para poder realizar la comparación con cada una. Así mismo, para la cuantía solo cumplió con la mínima que es el $1 \%$ pero en los análisis estáticos y dinámicos se logra mostrar su variación y sus fuerzas de diseño de cada norma.

a)

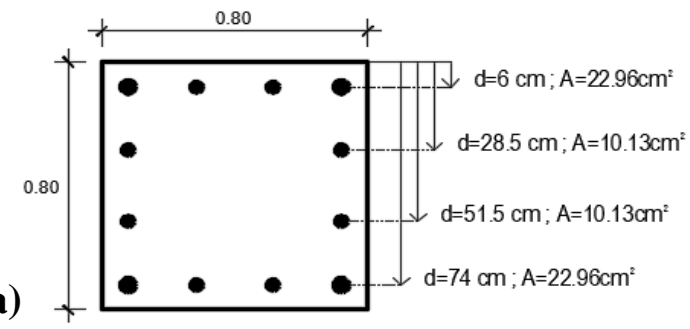

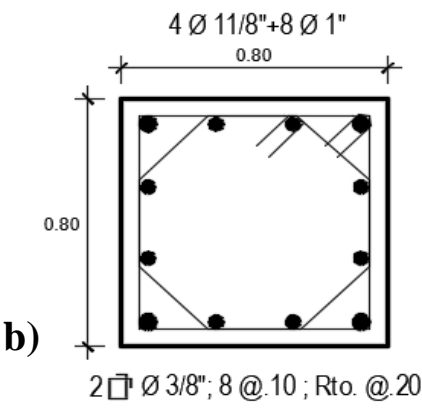

Figura 14. a) Distribución en la columna y b) Acero en la columna. Fuente: los autores

Las vigas si mostraron modificaciones considerando que la norma 2016 y 2018 son iguales para estructuras regulares, a diferencia de las estructuras irregulares que si presentan cambios en cada actualización. Las estructuras regulares según el análisis estático mediante periodos modales en los pórticos más esforzados tienen una diferencia de $0,9 \mathrm{~cm}^{2}$ de la norma 2003 a 2016 para ambos momentos positivos y negativos, por tal motivo se cambió de sección de acero 5/8" a 3/4" solo para momentos positivos, ya que para momentos negativos se considera una sección de acero la cual cubre los momentos del 2003 y 2016 como se muestra en la Figura 15.

a)

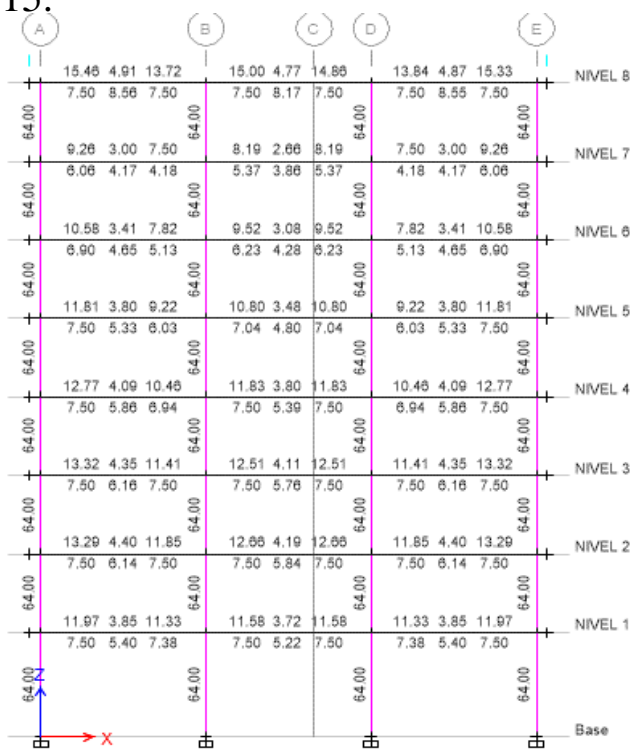

b)

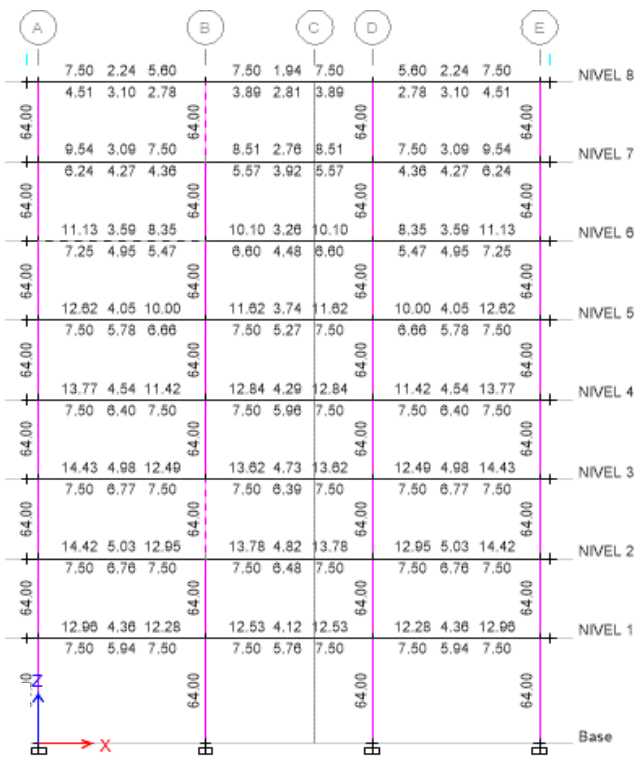

Figura 15. Área de acero requerido de la estructura regular de los pórticos (2-2) y (C-C). a) E.030 2003. b) E.030 2016-2018. Fuente: los autores 
Las estructuras regulares según el análisis dinámico en los pórticos más esforzados tienen una diferencia de $0,8 \mathrm{~cm}^{2}$ de la norma 2003 a 2016 para ambos momentos positivos y negativos, pero a pesar de la mínima diferencia con el análisis estático las secciones comerciales de acero se tendrán la misma distribución como se muestra en la Figura 16.

a)

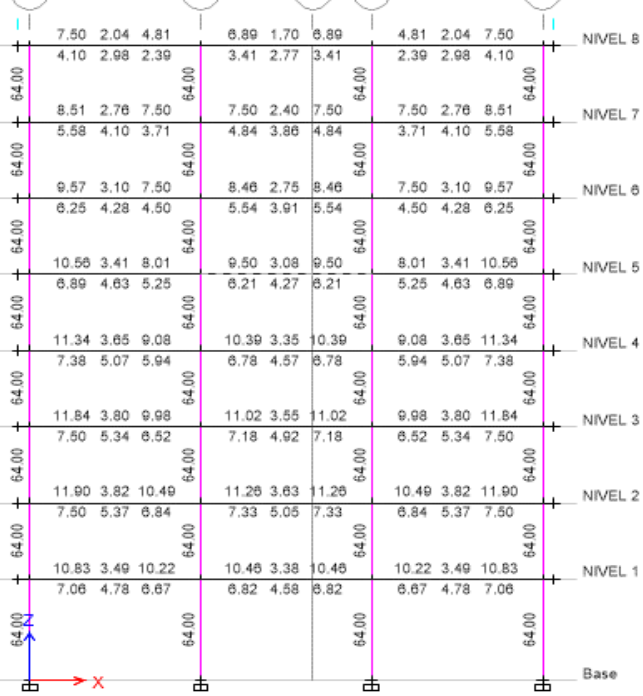

b)

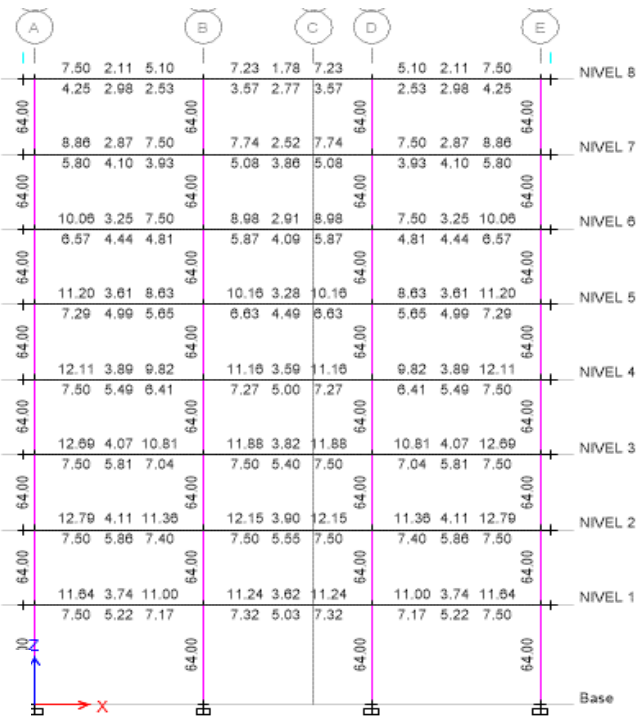

Figura 16. Área de acero requerido de la estructura regular de los pórticos (2-2) y (C-C). a) E.030 2003. b) E.030 2016-2018. Fuente: los autores

Las estructuras irregulares según el análisis dinámico en los pórticos más esforzados requieren área de acero de $16,70 \mathrm{~cm}^{2}, 24,26 \mathrm{~cm}^{2}, 20,43 \mathrm{~cm}^{2}$ según la norma del 2003, 2016 y 2018 en la dirección más desfavorable $Y$ - $Y$ como se muestra en la Figura 17 y 18 . Validando los resultados del análisis comparativo, así como los efectos que pueden ocasionar las actualizaciones de las normativas y resultado del incremento de las cortantes en la base para la norma del 2003 al 2016 y la reducción para la norma del 2016 al 2018.

a)

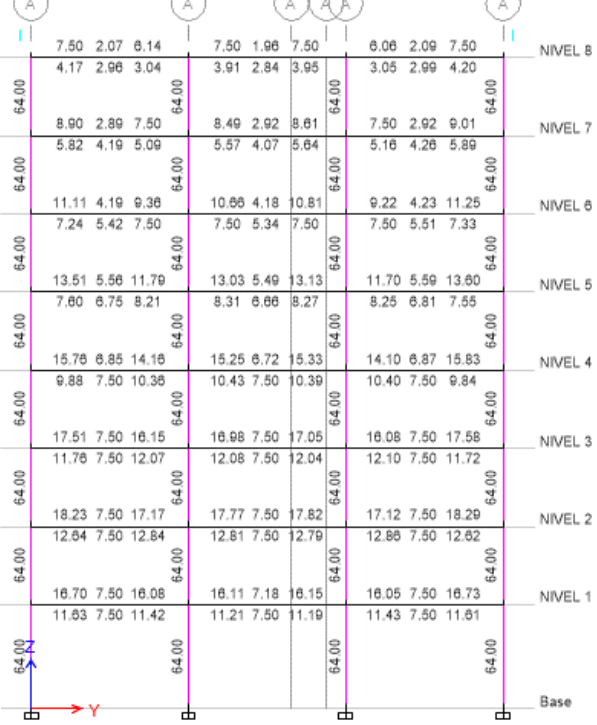

b)

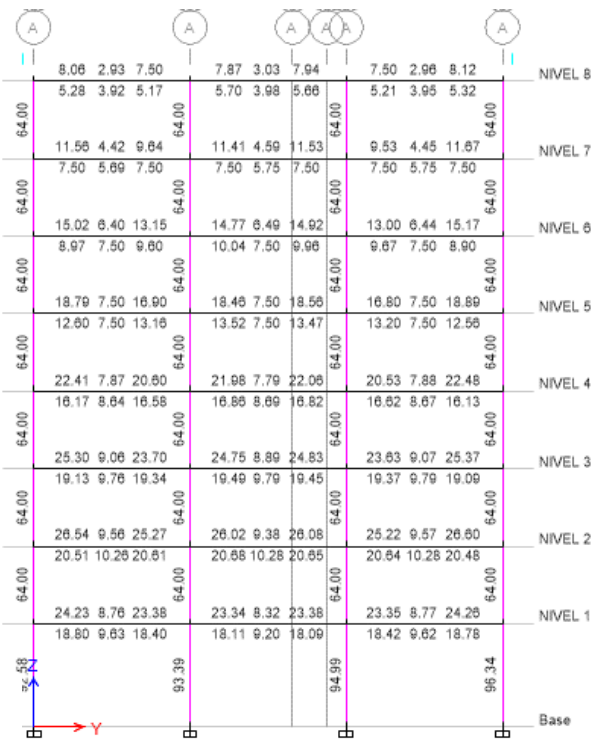

Figura 17. Área de acero requerido de la estructura irregular de los pórticos (A-A) en la dirección $Y-Y$. a) E.030 2003, b) E.030 2016. Fuente: los autores

Revista Gaceta Técnica. Artículo de Investigación. 23(1), 48-71, enero-julio, 2022 
ANÁLISIS COMPARATIVO DEL DISEÑO ESTRUCTURAL DE UNA EDIFICACIÓN REGULAR E IRREGULAR DE OCHO NIVELES EN SISTEMA DE PÓRTICOS APLICANDO LA NORMA E.030 2003, 2016 Y 2018 DISEÑO SISMORRESISTENTE EN LA CIUDAD DE LIMA

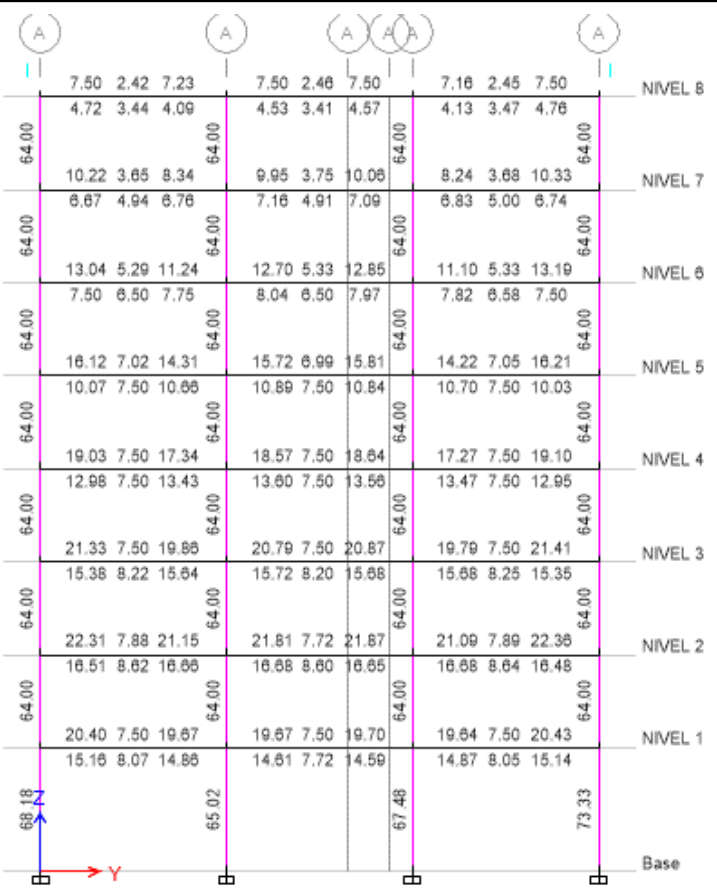

Figura 18. Área de acero requerido de la estructura irregular de los pórticos (A-A) en la dirección Y-Y según E.030 2018. Fuente: los autores

\section{Cimentaciones}

En el estudio no se considera zapatas aisladas esquineras y medianeras, por lo que convencionalmente no se utiliza en este tipo de proyectos, las cimentaciones están conformadas por zapatas aisladas centradas o concéntricas con un espesor de $80 \mathrm{~cm}$ y las secciones como se muestra en la Figura 19 según el análisis. Las cimentaciones son afectadas y generan cambios por las fuerzas sísmicas, en las estructuras regulares su espaciamiento cambia hasta $\pm 3 \mathrm{~cm}$ y las estructuras irregulares hasta $\pm 5 \mathrm{~cm}$ según la E.030 2003, 2016 y 2018.
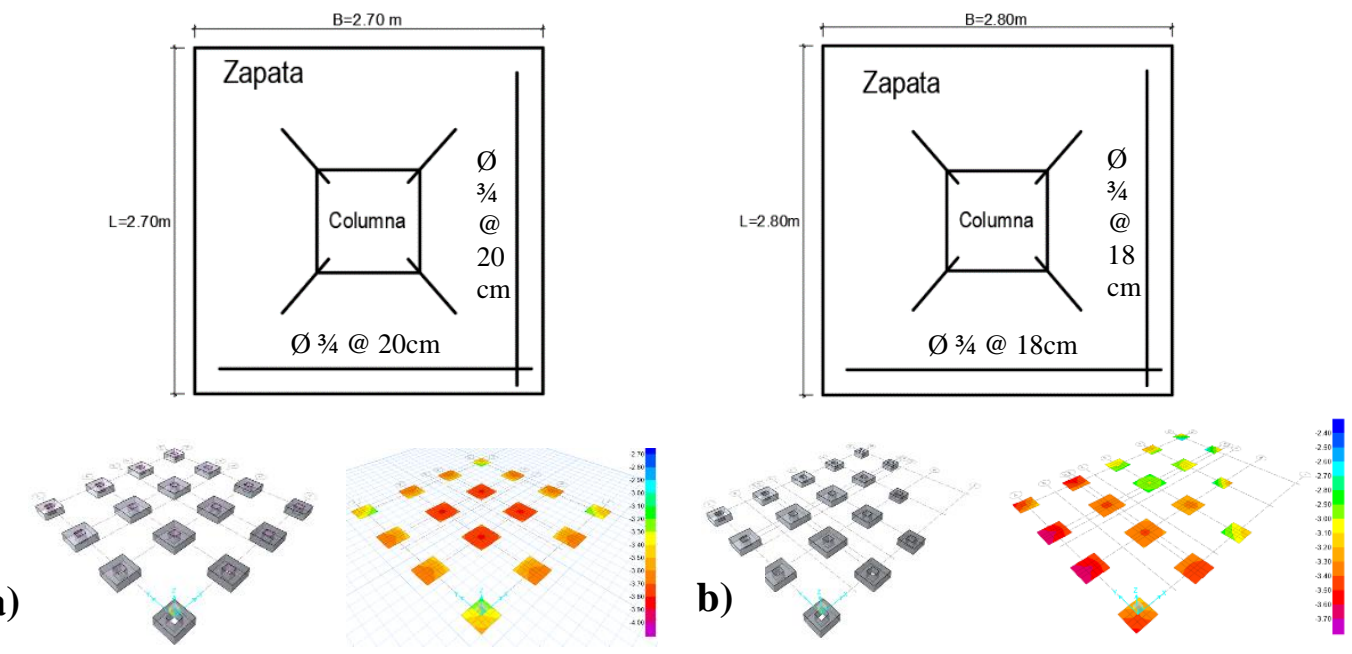

Figura 19. Zapatas aisladas centradas: a) Estructura regular ejes (2-C), b) Estructura irregular ejes (2-B). Fuente: los autores 


\section{CONCLUSIONES}

La estructura regular presenta una variación máxima de periodos fundamentales de 17,61\% comparando el periodo modal y alternativo, así mismo, la estructura irregular en la dirección $X$ - $X$ de $30,42 \%$ y en $Y$ - $Y$ de $27,27 \%$ comparando el periodo normativo y alternativo. El cotejo se realizó considerando que las normas del 2016 y 2018 son iguales para estructuras regulares con una variación de cortantes de 6,17\% entre periodos y 12,50\% según la norma del 2003 en comparación del 2016-2018. En consecuencia, la fuerza lateral según el periodo normativo tendrá un incremento de 17,16\% en el último nivel, variación de 13,59\% de distorsión en el tercer nivel y la fuerza de acuerdo al periodo modal de $-1,62 \%$ para el ultimo nivel, incremento de $22,68 \%$ en el penúltimo nivel y una variación de 13,12\% de distorsión en el tercer nivel según la norma del 2003 en comparación del 2016-2018. El análisis dinámico para estructuras regulares tiene un incremento en los espectros de diseño en 12,20\% y un ligero descenso de la curvatura para periodos largos. Por tanto, incremento de distorsión máxima en el tercer nivel de 13,16\% según la norma del 2003 en comparación del 2016-2018.

Las fuerzas máximas de diseño son de los pórticos (2-2) y (C-C), según las fuerzas estáticas del periodo modal obteniendo una variación de 11,96\%, 12,92\% y 12,62\% de fuerzas axiales, cortante y momentos flectores respectivamente y para las fuerzas dinámicas con un $12,5 \%$ en fuerzas axiales, cortantes y momentos flectores, reflejando esta variación en el diseño estructural de las vigas con una variación de $0,9 \mathrm{~cm}^{2}$ y $0,8 \mathrm{~cm}^{2}$ en área de acero del análisis estático y dinámico correspondientemente según la norma del 2003 en comparación del 20162018. Por otro lado, la estructura irregular según la norma 2003 presenta irregularidad de geometría vertical y esquina entrante, la norma del 2016 rigidez - piso blando y torsión y la norma del 2018 geometría vertical y torsión en planta como en altura respectivamente. Generando variación directa a las cortantes estáticas, espectros de diseño, distorsión y fuerzas máximas de diseño en 50\% entre la norma del 2003 y 2016, así mismo una variación de 16,67\% entre la norma del 2016 y 2018 para ambas direcciones, reflejando esta variación en el diseño estructural de las vigas con área de acero de $16,70 \mathrm{~cm}^{2}, 24,26 \mathrm{~cm}^{2}, 20,43 \mathrm{~cm}^{2}$ según la norma del 2003, 2016 y 2018 en la dirección más desfavorable $Y-Y$ del pórtico (A-A).

La variación que existe en fuerzas estáticas comparando con las fuerzas dinámicas en la dirección $Y-Y$ de la norma 2018 tiene una variación de 13,53\%, 5,65\% y 4,55\% de fuerzas axiales, cortante y momentos flectores respectivamente, fuerzas que dependen de periodos modales. Por ello, su variación es mínima a comparación de periodos normativos o alternativos en donde su variación será más crítica, por consecuente este tipo de estructuras 
ANÁLISIS COMPARATIVO DEL DISEÑO ESTRUCTURAL DE UNA EDIFICACIÓN REGULAR E IRREGULAR DE OCHO NIVELES EN SISTEMA DE PÓRTICOS APLICANDO LA NORMA E.030 2003, 2016 Y 2018 DISEÑO SISMORRESISTENTE EN LA CIUDAD DE LIMA

con irregularidad de geometría vertical y torsión se puede diseñar según el análisis estático considerando periodos modales. El diseño estructural se realizó de acuerdo a las normas peruanas, por lo que estas son basadas a los criterios estructurales de la norma del ACI logrando comprobar la similitud que existe en los resultados y la confiabilidad que brinda para el diseño.

\section{RECOMENDACIONES}

Es importante analizar cada parámetro sísmico y sus modificaciones desde el factor de zonificación que sigue en cambio por los estudios amplios de peligro sísmico, factor de amplificación y los cambios por los espectros de desplazamientos y el factor de suelo que ya no mide cuanto amplifica un suelo sino la diferencia de estar en un suelo bueno o malo. Es recomendable utilizar periodos alternativos y normativos a diferencia de periodos modales para estructuras regulares, y en estructuras irregulares lo más adecuado es utilizar periodos alternativos o modales, a diferencia de utilizar periodos normativos por lo que estas las esforzaran más estructuralmente como se puede ver en el análisis.

Se recomienda además, utilizar la norma del 2016-2018 para estructuras regulares, considerando que la variación sea mínima, pero será más conservadora y las estructuras con irregularidad de geometría vertical y torsión se debe diseñar con la norma del 2016 mediante el análisis dinámico o mediante el análisis estático utilizando periodos modales. La importancia de identificar las irregularidades de una estructura, la cual se reflejará en desplazamientos y fuerzas internas mayores, siendo estas favorables para el diseño estructural con mayor resistencia y rigidez en las estructuras.

\section{REFERENCIAS}

[1] J. Yepez, "Desempeño sismico de edificaciones esenciales," Propues. Vis. 2000, no. 65, pp. 65-86, 1996.

[2] C. Condori y H. Tavera, “Areas probables de ruptura sísmica en el borde occidental del perú, a partir de la variación del parámetro 'b,"” Soc. Geol. del Perú SGP, vol. 36, no. 1997, pp. 23-36, 2006.

[3] N.T.E, "Norma técnica de edificación," Sencico, pp. 1-45, 2003.

[4] R.N.E, “Decreto supremo n ${ }^{\circ}$ 002-2014-vivienda," Sencico, 2016.

[5] R.N.E, “RM-355-2018-VIVIENDA (2).pdf,” Sencico, 2018.

[6] S. Hampshire De C. Santos, L. Zanaica, C. Bucur, and S. De Souza Lima, "Comparative Study of Codes for Seismic Design of Structures," Math. Model. Civ. 
Eng., vol. 9, no. 1, pp. 1-12, 2013.

[7] J. L. C. Aedo and J. E. A. Hurtado, "Peligro sísmico en el perú," Peligro Sism. En El Peru, no. 1, p. 19, 1993.

[8] C. Aksoylu, A. Mobark, M. H. Arslan, and I. H. Erkan, "A comparative study on ASCE 7-16, TBEC-2018 and TEC-2007 for reinforced concrete buildings," Rev. la Constr., vol. 19, no. 2, pp. 282-305, 2020.

[9] C. Zajac and D. Todd, "A Comparative Analysis for Base Shear Calculations between Six Countries With Moderate Seismic Activity,” AEI, pp. 567-580, 2015.

[10] R. Salinas Basueldo, "Fundamentos Del Analisis Dinamico De Estructuras," Univ. Nac. ed Ing., pp. 1-24, 2013.

[11] A. Jorge, M. Jorge, M. Jose, and H. Carlos, “Avances en la microzonificacion sismica de lima, perú," CISMID, vol. 1, p. 12, 1991.

[12] H. J. Maco Sarmiento, “Análisis y diseño de una edificación multifamiliar de siete pisos con muros de ductilidad limitada (tesis de titulación)," Pontif. Univ. Católica del Perú, 2014.

[13] NTE E.060, Reglamento Nacional de Edificaciones. Norma E.060 Concreto Armado. 2016.

[14] NTE E.050, "Suelos Y Cimentaciones,” El Peru., pp. 22-68, 2018.

[15] J. Landingin, H. Rodrigues, H. Varum, A. Arêde, and A. Costa, "Comparative Analysis of RC Irregular Buildings Designed According to Different Seismic Design Codes," Open Constr. Build. Technol. J., vol. 7, no. 1, pp. 221-229, 2014.

[16] R. Medina, "Determinación del nivel de desempeño de un edificio habitacional estructurado en base a muros de hormigón armado y diseñado según normativa chilena," Obras y Proy., no. 23, pp. 63-77, 2018. 